ARTICLE

https://doi.org/10.1038/s41467-019-10755-3

\title{
PRC1 collaborates with SMCHD1 to fold the X- chromosome and spread Xist RNA between chromosome compartments
}

Chen-Yu Wang (1) 1,2, David Colognori ${ }^{1,2,3}$, Hongjae Sunwoo (iD 1,2,3, Danni Wang (i) ${ }^{1,2}$ \& Jeannie T. Lee (i) 1,2

$\mathrm{X}$-chromosome inactivation triggers fusion of $\mathrm{A} / \mathrm{B}$ compartments to inactive $\mathrm{X}(\mathrm{Xi})$-specific structures known as $\mathrm{S} 1$ and $\mathrm{S} 2$ compartments. SMCHD1 then merges $\mathrm{S} 1 / \mathrm{S} 2 \mathrm{~s}$ to form the $\mathrm{Xi}$ super-structure. Here, we ask how S1/S2 compartments form and reveal that Xist RNA drives their formation via recruitment of Polycomb repressive complex 1 (PRC1). Ablating Smchd1 in post- $\mathrm{XCl}$ cells unveils S1/S2 structures. Loss of SMCHD1 leads to trapping Xist in the S1 compartment, impairing RNA spreading into S2. On the other hand, depleting Xist, PRC1, or HNRNPK precludes re-emergence of S1/S2 structures, and loss of S1/S2 compartments paradoxically strengthens the partition between $X_{i}$ megadomains. Finally, $X_{i}-$ reactivation in post-XCl cells can be enhanced by depleting both SMCHD1 and DNA methylation. We conclude that Xist, PRC1, and SMCHD1 collaborate in an obligatory, sequential manner to partition, fuse, and direct self-association of $\mathrm{Xi}$ compartments required for proper spreading of Xist RNA.

\footnotetext{
${ }^{1}$ Department of Molecular Biology, Massachusetts General Hospital, Boston, MA, USA. ${ }^{2}$ Department of Genetics, The Blavatnik Institute, Harvard Medical School, Boston, MA, USA. ${ }^{3}$ These authors contributed equally: David Colognori, Hongjae Sunwoo. Correspondence and requests for materials should be addressed to J.T.L. (email: lee@molbio.mgh.harvard.edu)
} 
M ammalian chromosomes show a distinct topological organization. At one level, chromosomes are partitioned into A and B compartments in interphase nuclei, with gene-rich, active chromatin partitioned into the A compartment, and gene-poor, inactive chromatin into the B compartment ${ }^{1}$. At a finer level, chromosomes are segregated into "topologically associated domains" (TADs) on a megabase scale $^{2,3}$. Genetic elements interact frequently within TADs, but are insulated from each other between TADs. How these chromosome structures are constructed is an area of intense investigation. Evidence suggests that TAD formation requires two architectural proteins-cohesin and CCCTC-binding factor $(\mathrm{CTCF})^{4,5}$, with cohesin progressively extruding chromatin loops until reaching a pair of convergent CTCF sites at defined TAD borders $^{6,7}$.

Much less is known about how chromosome compartments are constructed. As compartments persist in the absence of CTCF or cohesin $^{4,5,8,9}$, compartmentalization occurs via a mechanism distinct from that of TADs. An important feature of chromosome compartments is their correlation with chromatin states ${ }^{1,6}$. Thus, it is thought that compartments are driven by self-association between the same type of chromatin, possibly in a biophysical process known as "liquid-liquid phase separation" (LLPS) ${ }^{10,11}$. Indeed, many chromatin-associated factors phase separate ${ }^{10-13}$. However, evidence that these factors are required for forming large-scale chromosome compartments is currently lacking.

$\mathrm{X}$-chromosome inactivation (XCI), the mechanism by which mammals balance dosage of X-linked genes between males and females, is a powerful model to study chromosome architecture $^{14-19}$. During XCI, the inactive $\mathrm{X}$ chromosome (Xi) undergoes massive reconfiguration into a unique structure distinct from the active $\mathrm{X}$ chromosome $(\mathrm{Xa})$. For instance, the $\mathrm{Xi}$ acquires two large interacting domains separated by a tandem repeat locus, $D x z 4^{6,20-22}$. Concurrent with formation of these "megadomains" is global attenuation of TADs ${ }^{20-22}$. Central to this process is Xist, a long noncoding RNA that spreads along the $\mathrm{Xi}$, recruiting silencing factors and inducing a global suppression of TADs ${ }^{21-24}$

Structural reorganization of the $\mathrm{Xi}$ also occurs at the level of compartments. The Xi is unique in being "compartment-less," without the $\mathrm{A} / \mathrm{B}$ compartmentalization that characterizes all mammalian chromosomes ${ }^{22}$. By ablating an Xi-enriched protein, structural maintenance of chromosomes hinge domain containing 1 (SMCHD1 $)^{25-27}$, in pre-XCI cells and observing effects during de novo XCI, we recently identified a hidden layer of Xi organization-S1/S2 compartments ${ }^{28}$. This Xi-specific structure marks a transition in the progression from $\mathrm{A} / \mathrm{B}$ compartments to the "compartment-less" structure. We proposed a stepwise folding origami model, in which $\mathrm{A} / \mathrm{B}$ compartments are conjoined into S1/S2 compartments, which are then merged by SMCHD1 to create the Xi super-structure. During de novo XCI, failure to merge compartments results in compromised spreading of Xist RNA, segmental erosion of heterochromatin, and failed silencing of $\sim 43 \%$ of genes normally subject to $\mathrm{XCI}^{28}$. Thus, S1/S2 compartments provide an example where the dynamic control of compartment structure contributes to gene regulation. Although it is clear that the merging of S1/S2 compartments relies on SMCHD1, the mechanism underlying their formation is presently unknown.

Here we investigate the molecular requirements of the stepwise folding mechanism. We show that merged Xi compartments can be unfolded. Ablating Smchd1 in post-XCI cells causes reemergence of transitional S1/S2 compartments. SMCHD1 deficiency also traps Xist RNA in the S1 compartment, leading to aberrant enrichment of $\mathrm{H} 3 \mathrm{~K} 27 \mathrm{me} 3$ on the Xi. Furthermore, depleting Xist, heterogeneous nuclear ribonucleoprotein $K$
(HNRNPK), or polycomb repressive complex 1 (PRC1) prevents S1/S2 compartmentalization. These results reveal a dependence on PRC1 for creation of S1/S2 structures and a role of SMCHD1 in facilitating the spreading of Xist RNA between S1/S2 chromosome compartments.

\section{Results}

Post-XCI Smchd1 ablation unveils S1/S2 compartments. We began by asking if SMCHD1 is required once the $\mathrm{Xi}$ is already established. We disrupted Smchd1 in mouse embryonic fibroblasts (MEFs), a cell type in which XCI is completed (Supplementary Fig. 1a-c). To probe three-dimensional (3D) organization of the Smchd1-/- $\mathrm{Xi}$, we performed allele-specific in situ Hi-C on wild-type (WT) and Smchd1-/- clones, taking advantage of $>600,000$ sequence polymorphisms between the Xa of Mus castaneus (cas) origin and the Xi of Mus musculus (mus) origin to call alleles ${ }^{29}$. As predicted, the Xa's of $S m c h d 1^{-1-}$ and WT clones showed no significant differences between one another (Supplementary Fig. 2a).

On the other hand, the $\mathrm{Xi}$ showed striking differences. Whereas the WT $\mathrm{Xi}$ exhibited two megadomains separated by the boundary element, Dxz4 (Fig. 1a, left) $6,17,20-22,30$, the Smchd $1^{-1-} \mathrm{Xi}$ developed a heterogeneous pattern within each megadomain (Fig. 1a, right). Its Pearson correlation map showed a distinct checkerboard pattern suggesting smaller compartment structures appearing under the megadomains (Fig. 1b, right). This pattern was distinct from the A/B compartmental pattern of the Xa (Supplementary Fig. 2a, bottom). Principal component analysis (PCA) verified the changes in Smchd $1^{-1-}$ cells. Indeed, principal component 1 (PC1) showed that the Smchd1-1- Xa retained the $\sim 63 \mathrm{~A} / \mathrm{B}$ compartments that characterize the WT Xa $(r=0.94)$ (Supplementary Fig. 2b), but the Smchd1 ${ }^{-/}-\mathrm{Xi}$ lost the compartment-less character of the WT Xi. Instead of the two large compartments that characterize the WT Xi, the mutant $\mathrm{Xi}$ exhibited $\sim 25$ compartments averaging $4.1 \mathrm{Mb}$ in size (Fig. 1c). Notably, the 25 compartments were highly similar to S1/S2 compartments that occur transiently during de novo XCI $(r=$ 0.84, Fig. 1d, Supplementary Fig. $3 \mathrm{a}-\mathrm{c})^{28}$. Thus, SMCHD1 loss is sufficient to unmask underlying S1/S2 compartments, and the folding of the $\mathrm{Xi}$ compartments appears to be reversible.

Chromatin compartments generally align with epigenetic landscapes $1,6,31,32$. Accordingly, on the Xa, A compartments correlated with active H3K4me3 marks in both WT and Smchd $1^{-1-}$ cells $(r=0.52)$ (Supplementary Fig. 2b). On the Smchd $1^{-1-} \mathrm{Xi}$, the re-emergent S1/S2 compartments remained correlated with the H3K27me3 density $(r=0.77)$ and Xist RNA binding $(r=0.70)$ that normally typifies the $\mathrm{Xi}$ (Fig. 1c, Supplementary Fig. 3d). Consistent with its role in merging S1/S2s ${ }^{28}$, analysis of SMCHD1 binding patterns in MEFs indicated localization across both $\mathrm{S} 1$ and S2 domains (Fig. 1c, Supplementary Fig. 3d). Together, these data show that Smchd1 ablation unveils the transitional S1/S2 conformation, where Xistrich (S1) and Xist-poor (S2) chromatin spatially segregate. We conclude that SMCHD1 is actively required to maintain the merging of S1/S2 compartments.

Interestingly, although S1/S2 compartments reappeared following Smchd1 ablation, the Smchd1 $1^{-I-} \mathrm{Xi}$ did not lose its overall bipartite structure. Indeed, whereas PC1 reflected S1/S2 compartments, principal component 2 (PC2) captured the two megadomains (Fig. 1c). These megadomains were also discernible on the contact heatmap of the Smchd $1^{-1-} \mathrm{Xi}$, even though the $\mathrm{Xi}$ adopted a checkerboard pattern (Fig. 1a, b). These observations suggest that SMCHD1 deficiency does not eradicate the overall bipartite structure and merely unmask an S1/S2 organization merged on the WT Xi. S1/S2 compartments and megadomains 
a

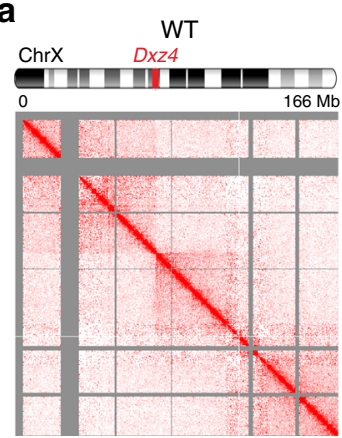

b

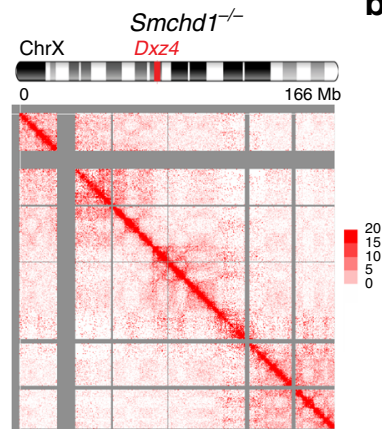

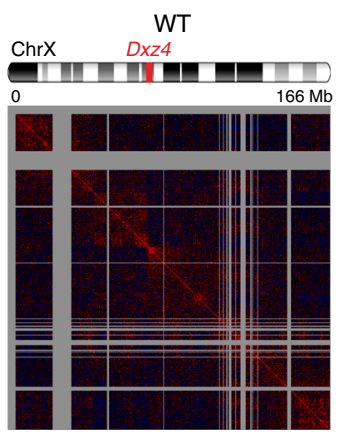

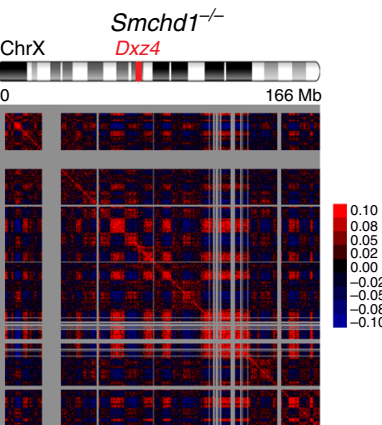

C

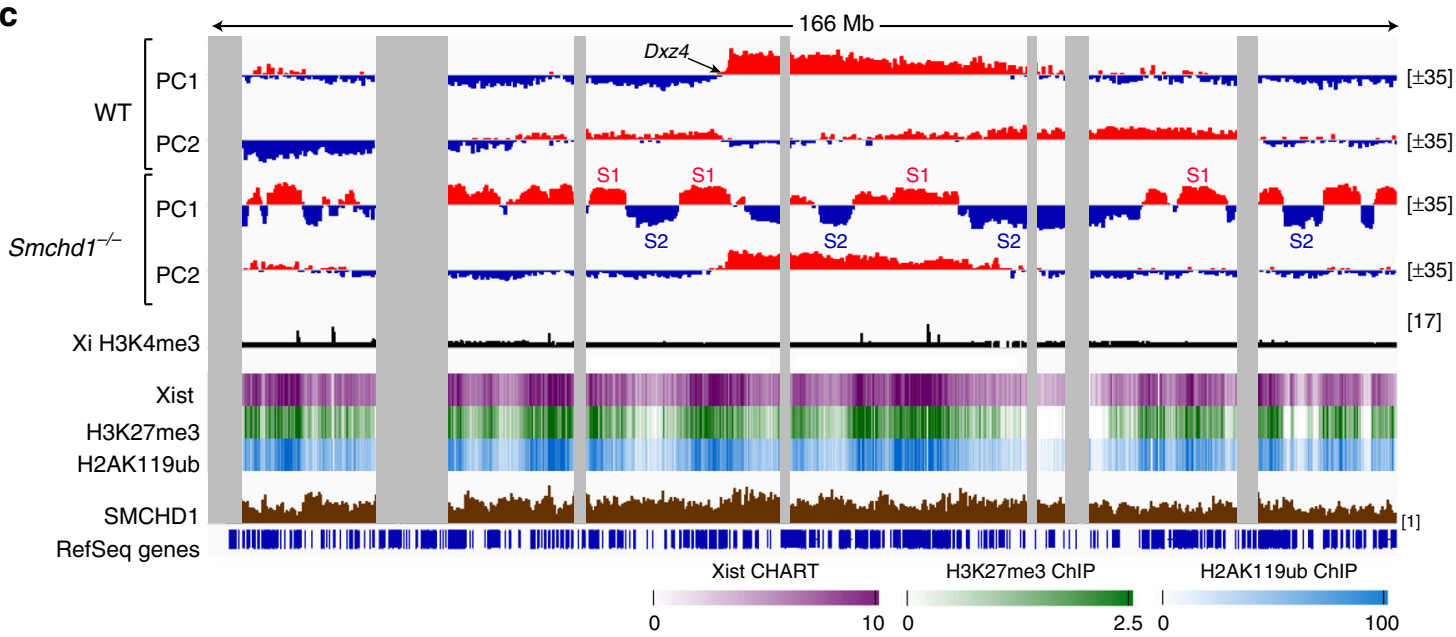

d

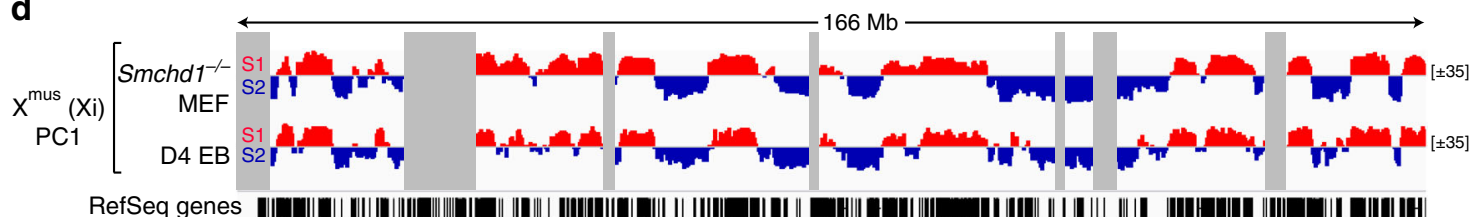

e

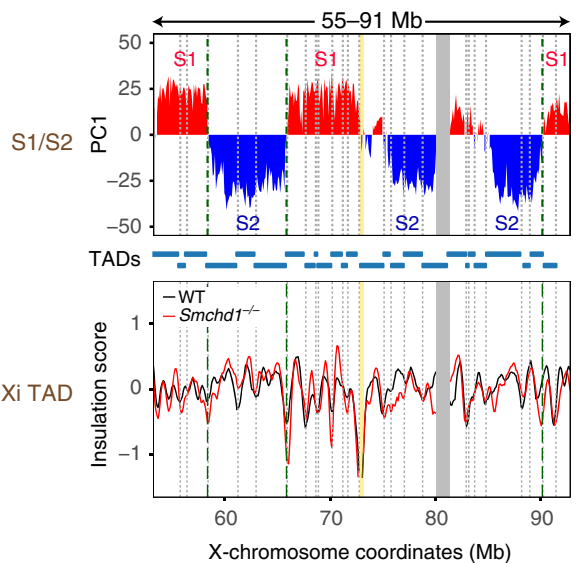

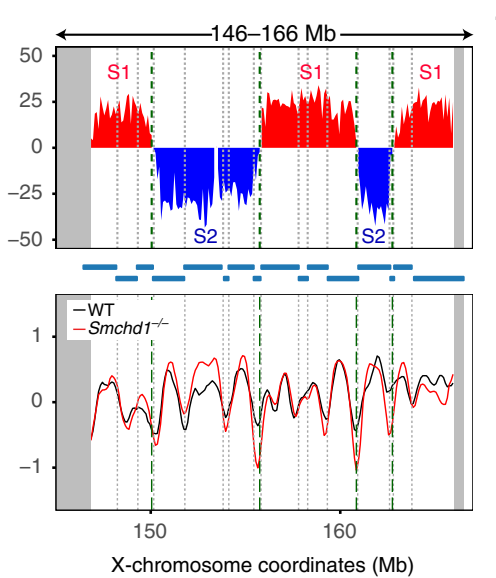

f $\quad \mathrm{S} 1 / \mathrm{S} 2$ compartment borders

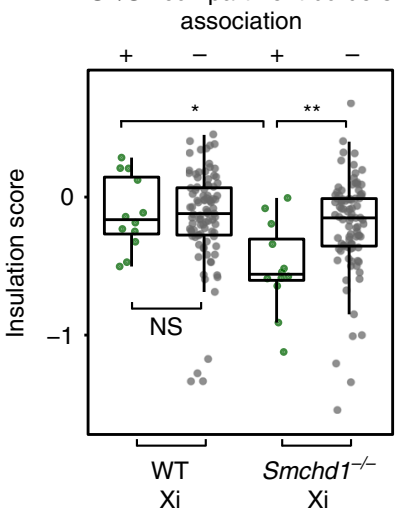

are therefore two different levels of $3 \mathrm{D}$ organization superimposed on the $\mathrm{Xi}$.

SMCHD1 is required to maintain Xi TAD attenuation. Chromosome conformation studies have provided two different views of TAD organization on the $\mathrm{Xi}$. Some studies indicated that TADs are dissolved during $\mathrm{XCI}^{21,22}$. However, the others using improved $\mathrm{Hi}-\mathrm{C}$ methods have suggested that TADs remain on the $\mathrm{Xi}$, albeit in an attenuated state $20,24,28,30$. One study from our lab furthermore showed that depleting SMCHD1 during de novo
XCI compromises TAD suppression ${ }^{28}$. Here we asked if ablating Smchd 1 in post-XCI cells causes a change to Xi TADs. Analysis of $\mathrm{Hi}-\mathrm{C}$ contact maps at $100-\mathrm{kb}$ resolution suggested that TADs also remained-in a weakened state-on the WT MEF Xi (Supplementary Fig. 4a). To quantify TAD strength, we computed insulation scores, a parameter that quantifies the frequency of chromatin interactions across a genomic region ${ }^{22}$. The insulation scores of the WT Xi fluctuated at smaller amplitude (Supplementary Fig. 4a, b), resulting in a steeper cumulative distribution curve than those of the WT Xa (Supplementary Fig. 4c, left). However, their local minima (representing TAD boundaries) and 
Fig. 1 Depleting structural maintenance of chromosomes hinge domain containing 1 (SMCHD1) leads to reappearance of S1/S2 compartments in post-Xchromosome inactivation (XCl) cells. a Depth-corrected chromatin interaction maps of the inactive X chromosome (Xi) in wild-type (WT) and Smchd1-/mouse embryonic fibroblasts (MEFs) binned at 200-kb resolution. Gray-shaded areas, unmappable regions. Also see Supplementary Fig. 2a for the active $\mathrm{X}$ chromosome (Xa) maps. b The corresponding Pearson's correlation maps of the Xi in WT and Smchd1-/- MEFs binned at 200-kb resolution. Also see Supplementary Fig. 2a for the Xa maps. c Principal component 1 (PC1) and PC2 values of the Xi in WT and Smchd1-/- MEFs. Regions with positive PC1 values on the Smchd1-/- Xi represent the S1 (Xist-rich) compartment (red). Also shown are Xi-specific H3K4me3 chromatin immunoprecipitation followed by deep sequencing (ChIP-seq) peaks (Xmus, GSE33823), Xist CHART-seq (capture hybridization analysis of RNA targets with deep sequencing) (GSE48649), H3K27me3 ChIP-seq (GSE33823), H2AK119ub ChIP-seq (GSE107217), and SMCHD1 DNA adenine methyltransferase identification by sequencing (DamID-seq) (GSE99991) profiles in WT MEFs. Xist, H3K27me3, and H2AK119ub profiles were displayed as heatmaps, with scale bars shown below the tracks. Also see Supplementary Fig. $2 b$ for the PCs of the Xa. d PC1 values of the Xi in Smchd1-/- MEFs and embryoid bodies formed after 4 days of differentiation of female WT mouse embryonic stem cells (D4 EB, early XCI) (GSE99991). Regions with positive PC1 values represent the S1 (Xist-rich) compartment (red). e Comparison of the compartment profile (PC1) (top) of the Smchd1 ${ }^{-}-X_{i}$ and the topologically associated domain (TAD) insulation profiles (bottom) of the WT (black) and Smchd1-/- Xi (red) at two representative X-linked regions. TADs (as defined in Dixon et al. ${ }^{2}$ ) were depicted as blue bars between plots and as dashed lines (TAD boundaries) within each plot. Green dashed lines, the borders of S1/S2 compartments. Yellow-shaded area: Dxz4. Gray-shaded areas, unmappable regions. $\mathbf{f}$ Box plots comparing the insulation scores of the TAD boundaries associated with borders of S1/S2 compartments (green) and the other TAD boundaries (gray) on the Xi. Note that lower insulation scores indicate stronger insulation effects. $P$ values are given by the Wilcoxon's rank-sum test. NS, not significant $(P>0.05)$. ${ }^{\star} P=0.0018$; ${ }^{\star \star} P=0.0015$. Midline, median. Top and bottom of the box, first and third quartile. Whiskers, extension from the top or bottom to the furthest datum within 1.5 times the interquartile range

maxima often coincided (Supplementary Fig. 4a, b), consistent with the idea that Xi TADs are attenuated.

When Smchd1 was ablated, however, there was an overall strengthening of Xi TADs (Supplementary Fig. 4a), indicating that SMCHD1 loss in the post-XCI stage also partially restores TADs. Insulation analysis revealed a greater fluctuation of the Smchd1 ${ }^{-1-}$ Xi (Fig. 1e, Supplementary Fig. 4a, b). In support, the insulation scores of the Smchd1 $1^{-/} \mathrm{Xi}$ exhibited a shallower cumulative distribution than those of the WT Xi (Supplementary Fig. 4d, right), consistent with stronger TADs. There was also an Xi-specific increase of TAD boundary strength (Supplementary Fig. 4 e) and greater correlation of Xi TAD organization to the Xa (Supplementary Fig. 4f) in Smchd1 $1^{-/}$MEFs. We conclude that SMCHD1 is required to suppress TADs during both the establishment and maintenance phases of XCI.

TAD boundaries are known to demarcate functional chromatin domains ${ }^{33,34}$. Interestingly, most (92\%) S1/S2 borders coincided with TAD boundaries (Fig. 1e, Supplementary Fig. 5). Moreover, the TAD boundaries that demarcate S1/S2 compartments tended to have stronger insulation effects (Fig. 1f). These findings indicate that TAD boundaries may play a role in demarcating the Xist-rich and Xist-poor compartments.

SMCHD1 and DNA methylation synergize in maintaining XCI. Because SMCHD1 deficiency during de novo XCI significantly affects $\mathrm{Xi}$ silencing $25,28,35,36$, we asked if depleting SMCHD1 would reactivate the $\mathrm{Xi}$ in post-XCI cells. We performed RNA-sequencing (RNA-seq) on two independent Smchd $1^{-1-}$ and two WT MEF clones. Interestingly, expression of $\mathrm{X}$-linked genes was mostly unchanged (Fig. 2a, Supplementary Fig. 6a, b). To examine the Xi specifically, we conducted allelespecific analysis on 216 genes subject to XCI in WT MEFs (216 genes passed our pipeline requirements) (Fig. 2b, Supplementary Data 1). Examining the fraction of $\mathrm{Xi}$ expression (\%mus) and cumulative distribution plots revealed no significant difference between WT and Smchd1 $1^{-1-}$ MEFs (Fig. 2c). Thus, Xi silencing was untouched by post-XCI SMCHD1 depletion. We conclude that losing SMCHD1 alone is not sufficient to reactivate $\mathrm{Xi}$ genes.

It is known that multiple repressive mechanisms collaborate to maintain $\mathrm{Xi}$ silencing ${ }^{37}$ and that reactivation requires perturbing multiple synergistic mechanisms ${ }^{21}$. Because DNA methylation has been identified as a major epigenetic mark to lock in the silent state $^{21,37,38}$, we treated Smchd1 ${ }^{-1-}$ cells with 5-aza-2'-deoxycytidine (Aza), a DNA-demethylating agent. Intriguingly, whereas Aza treatment caused only a mild Xi reactivation in WT MEFs
(Fig. 2d, left), it led to considerable Xi upregulation in Smchd1 $1^{-/-}$ MEFs, as demonstrated by a rightward shift of the \%mus cumulative distribution curve for $S m c h d 1^{-/-}$relative to WT cells, and also for dimethyl sulfoxide (DMSO) vs. Azatreated Smchd $1^{-/-}$cells (Fig. 2d, right panels). Heatmap analyses (Fig. 2e) and transcriptomic profiles (Fig. 2f) verified the $\mathrm{Xi}$ upregulation. By contrast, Aza treatment did not affect escapees, a subset of X-linked genes that resist XCI (Supplementary Fig. 6c 39,40 , Supplementary Data 1). These data argue that SMCHD1 and DNA methylation operate synergistically to maintain $\mathrm{Xi}$ silencing.

As only a fraction of $\mathrm{Xi}$ genes was destabilized, we investigated factors correlating with the differential response of $\mathrm{Xi}$ genes. Genes in S1 and S2 compartments responded similarly (Supplementary Fig. 6d). It is known that SMCHD1 is uniquely required for de novo silencing of a specific subset of X-linked genes-the "Class I" genes-but is dispensable for inactivating other X-linked genes, including the Class II and Class III genes (defined in Methods and listed in Supplementary Data 2) 28 . Intriguingly, Class I genes were generally more prone to reactivation (Fig. 2g, Supplementary Fig. 7), although not all Class I genes were reactivated and a small fraction of Class II and III genes was also destabilized. Thus, the class of genes that were more sensitive to SMCHD1 loss during de novo $\mathrm{XCI}^{28}$ is the same class that are more prone to reactivation in SMCHD1-deficient, post-XCI cells.

\section{Xist RNA is trapped in the S1 compartment in Smchd1 ${ }^{-/-}$} cells. We previously reported segmental erosion of $\mathrm{H} 3 \mathrm{~K} 27 \mathrm{me} 3$ domains on the Xi established without SMCHD1, most prominently in regions harboring derepressed Class I genes ${ }^{28}$. Here we asked if $\mathrm{H} 3 \mathrm{~K} 27 \mathrm{me} 3$ also became eroded in post-XCI cells depleted of SMCHD1. At the cytological level, Smchd $1^{-/-}$MEFs exhibited H3K27me3 foci co-localizing with Xist clouds (Fig. 3a, two Xist clouds seen because of tetraploidy), consistent with previous reports ${ }^{25,27}$, giving the appearance that $\mathrm{H} 3 \mathrm{~K} 27 \mathrm{me} 3$ on the $\mathrm{Xi}$ was not grossly affected. To determine if there might be regional erosion, we performed chromatin immunoprecipitation followed by deep sequencing (ChIP-seq) for H3K27me3. Gene bodies of escapees exhibited depletion of H3K27me3 in both WT and Smchd $1^{-T-}$ MEFs, as expected (Fig. 3b, Kdm5c, green-shaded area). On the other hand, Class I genes remained covered by this repressive mark, consistent with persistent gene silencing (Fig. 3b-d). Intergenic regions flanked by Class I genes, which also require SMCHD1 for de novo $\mathrm{H} 3 \mathrm{~K} 27 \mathrm{me}^{28}$, were also 
a

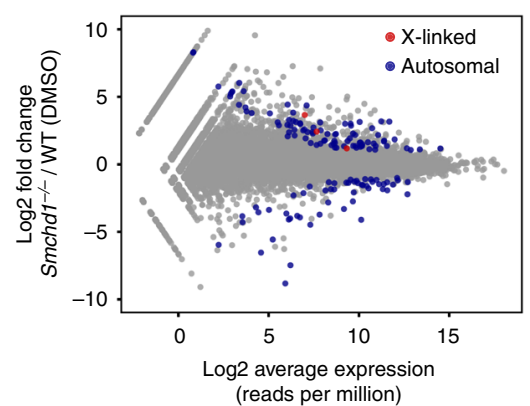

b

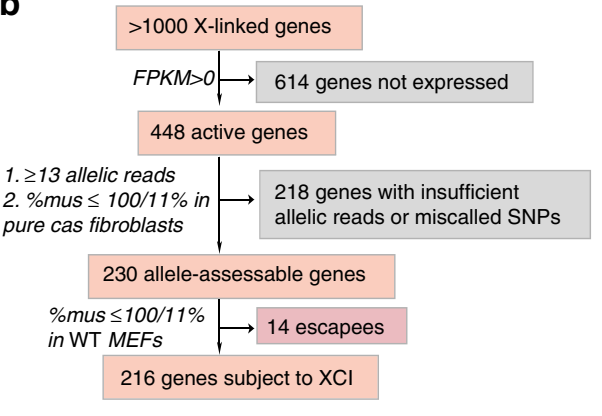

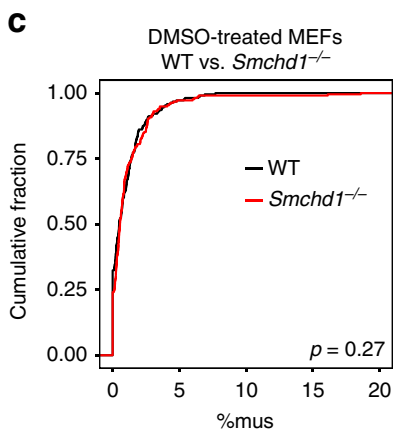

$\mathbf{e}$

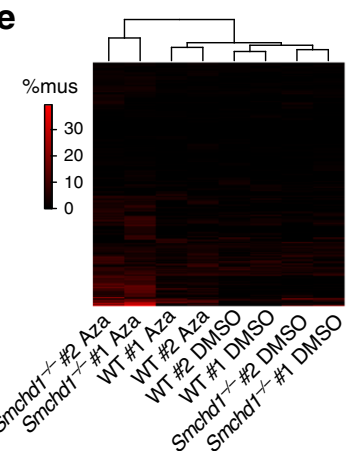

d

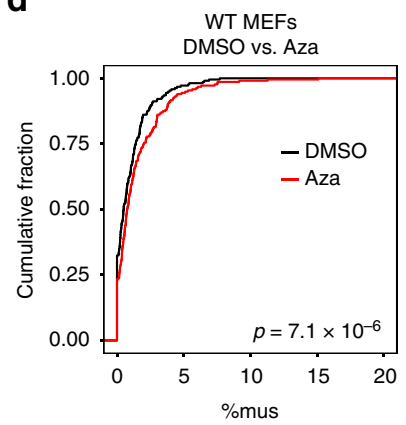

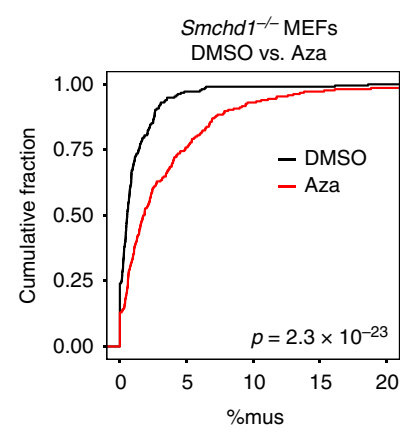

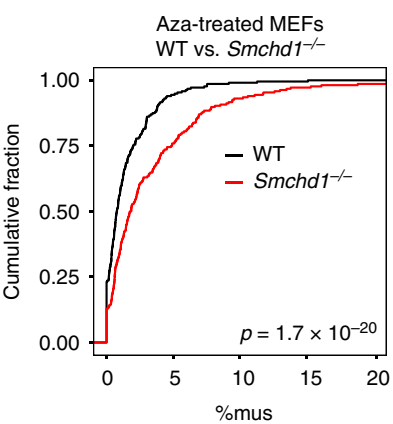

Aza-treated MEFs

f
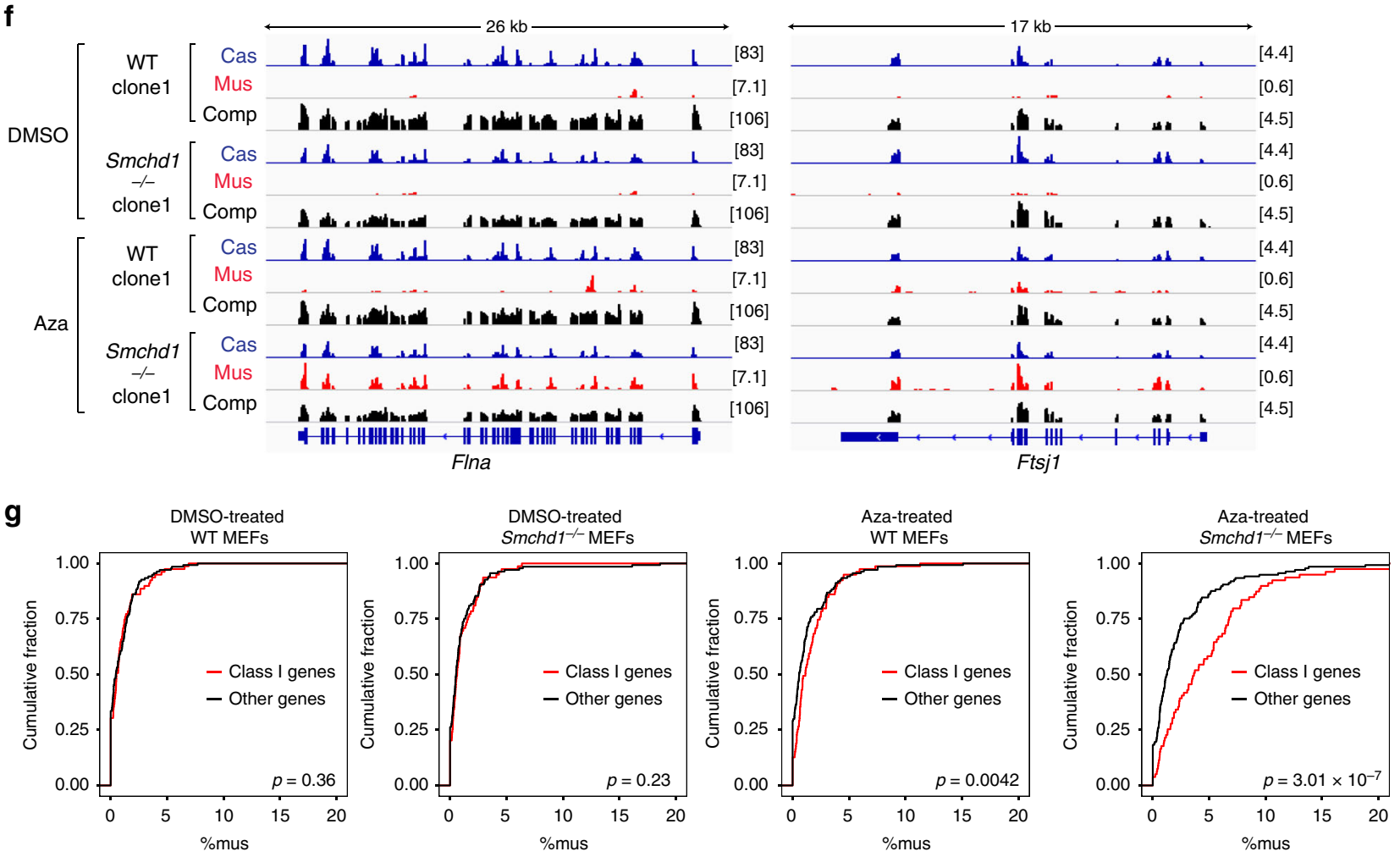

H3K27me3 enriched (Fig. 3b, c, e). Thus, SMCHD1 is not required to maintain $\mathrm{H} 3 \mathrm{~K} 27 \mathrm{me} 3$ in Class I regions. Other classes of X-linked regions also do not require SMCHD1 to maintain H3K27me3 (Fig. 3d, e; black dots).

Not only was SMCHD1 not required, but its depletion actually resulted in an overall increase of H3K27me3 enrichment on the $\mathrm{Xi}$ relative to the WT Xi (Fig. 3b-e). This enrichment was Xi specific (Fig. $3 \mathrm{f}$ ) and reproducible in two biological replicates (Supplementary Fig. 8a, b), and two independent normalization methods yielded the same conclusion (Supplementary Fig. 8c).

The finding was somewhat unexpected, as SMCHD1 generally promotes heterochromatin $28,35,36$, although one previous study suggested a similar increase of $\mathrm{H} 3 \mathrm{~K} 27 \mathrm{me}^{41}$. There was no copy number difference between WT and Smchd1 ${ }^{-/-} \mathrm{Xi}$, arguing against X-chromosome aneuploidy as a cause (Supplementary Fig. 9). To determine if aberrant $\mathrm{H} 3 \mathrm{~K} 27 \mathrm{me} 3$ accumulation is linked to the altered Xi structure, we investigated its correlation with S1/S2 compartments. As expected, H3K27me3 profiles correlated with $\mathrm{S} 1 / \mathrm{S} 2 \mathrm{~s}(r=0.92)$. Intriguingly, H3K27me3 was not homogeneously elevated on the Smchd $1^{-1-} \mathrm{Xi}$. Instead, this 
Fig. 2 Structural maintenance of chromosomes hinge domain containing 1 (SMCHD1) and DNA methylation synergistically maintain inactive $X$ chromosome (Xi) silencing. a An RNA-sequencing (RNA-seq) MA plot comparing the gene expression profiles of wild-type (WT) and Smchd1-/- mouse embryonic fibroblasts (MEFs) treated with dimethyl sulfoxide (DMSO). Gray dots, genes not differentially expressed. Blue dots, autosomal differentially expressed genes. Red dots, X-linked differentially expressed genes. b Workflow showing the identification of genes subject to X-chromosome inactivation $(\mathrm{XCl})$. Please see Supplementary Data 1 for the full list of genes subject to $\mathrm{XCl}$ and escapees. c Cumulative distribution plots (CDPs) of \%mus for genes subject to $\mathrm{XCl}$ in DMSO-treated WT and Smchd1-/- MEFs. $P$ values are given by the Wilcoxon's rank-sum test (paired, one-sided). d Cumulative distribution plots (CDPs) of \%mus for genes subject to XCI in DMSO/5-aza-2'-deoxycytidine (Aza)-treated WT and Smchd1-/- MEFs. $P$ values are given by the Wilcoxon's rank-sum test (paired, one-sided). e A heatmap showing the \%mus of genes subject to XCl in eight different RNA-seq datasets, with unsupervised hierarchical clustering accurately grouping clones with the same genotype and treatment together. $\mathbf{f}$ Allele-specific RNA-seq coverage tracks of Flna and Ftsj1, two representative genes subject to XCl. cas, cas-specific reads (active X chromosome; $\mathrm{Xa}$ ); mus, mus-specific reads (Xi). comp, all reads. To visualize rare mus reads originating from the $\mathrm{Xi}$, the scales of mus tracks were set differently from the cas tracks. For simplicity, only the minus strand was shown. $\mathbf{g}$ CDPs comparing \%mus of Class I genes vs. other genes subject to $\mathrm{XCl}$. $P$ values are given by the Wilcoxon's rank-sum test (unpaired, onesided). Please see Supplementary Data 2 for the full list of Class I genes defined previously 28

histone mark preferentially accumulated in the S1 compartment (Fig. 3g, h, Supplementary Fig. 8b), with the change in H3K27me3 ( $\Delta \mathrm{H} 3 \mathrm{~K} 27 \mathrm{me} 3)$ highly correlating with S1/S2s $(r=0.9)$.

Because Xist recruits PRC2 to the $\mathrm{Xi}^{42}$, we asked if changes in Xist spreading underlie elevated H3K27me3. Because ablating Smchd1 does not affect Xist expression in post-XCI cells (Fig. 4a), increased H3K27me3 on the Smchd1 $1^{-1-} \mathrm{Xi}$ was not caused by Xist upregulation. Although the Xist cloud morphology appeared cytologically normal in Smchd1 ${ }^{-/-}$cells (Fig. 3a), molecular differences might be visible by CHART-seq analysis (capture hybridization analysis of RNA targets with deep sequencing $)^{43}$, an epigenomic method to map Xist binding to chromatin at high resolution. Indeed, we previously showed a local defect of Xist spreading into Class I regions during de novo $\mathrm{XCI}^{28}$. By contrast, when SMCHD1 was depleted post$\mathrm{XCI}$, Xist binding to Class I regions was preserved (Fig. $4 \mathrm{~b}-\mathrm{d}$ ), consistent with $\mathrm{H} 3 \mathrm{~K} 27 \mathrm{me} 3$ remaining enriched at these regions (Fig. 3b-d). Intergenic regions flanked by two Class I genes also remained bound by Xist (Fig. $4 \mathrm{~b}, \mathrm{c}, \mathrm{e}$ ). There is, however, a striking overall redistribution of Xist along the Smchd1-/- Xi (Fig. 4f). While the Xi remained broadly enriched with Xist, we observed heightened peaks and deepened valleys on the Smchd $1^{-/}$Xi (Fig. 4f). This may not reflect experimental variation of CHART, as difference in Xist distribution appeared in both biological replicates, and CHART in WT and Smchd1 -/- MEFs seemed to have similar efficiency (Supplementary Figs. 8b, 10). To quantify changes in Xist binding, we subtracted WT Xist coverage from that of Smchd $1^{-/-}(\Delta \mathrm{Xist})$. Strikingly, the $\Delta$ Xist profile correlated with $\Delta \mathrm{H} 3 \mathrm{~K} 27 \mathrm{me} 3 \quad(r=0.88)$, suggesting that changes in Xist RNA localization likely underlie aberrant deposition of $\mathrm{H} 3 \mathrm{~K} 27 \mathrm{me} 3$. As expected, Xist density on the Smchd1-/- Xi correlated with S1/S2 compartments $(r=0.86)$.

However, we noticed that the increase in Xist density on the Smchd1-/- Xi occurred specifically in the S1 compartment, whereas there was an obvious depletion in S2 (Fig. 4f, g), resulting in a $\Delta$ Xist profile that correlated highly with alternating $\mathrm{S} 1 / \mathrm{S} 2$ structures $(r=0.8)$. This was intriguing, as the Xist locus resides in the $\mathrm{S} 1$ compartment ${ }^{28}$. As such, it is tempting to speculate that the spatial segregation between $\mathrm{S} 1$ and S2 compartments on the Smchd1-/- $\mathrm{Xi}$ may compromise Xist RNA spreading from S1 to S2, resulting in a trapping of Xist in S1. This aberrant accumulation would explain the heightened H3K27me3 enrichment in S1. Thus, Smchd1 ablation affects Xist spreading during both de novo $\mathrm{XCI}^{28}$ and the maintenance phase, but the effect is manifested differently during the two phases. In the former, Xist binding to Class I regions is compromised. In the latter, Xist can continue to spread across the S1 compartment (including Class I regions), but spreading into S2 is impaired.
Xist is required to form $\mathrm{S} 1 / \mathrm{S} 2$ compartments. Next, we turned attention to understanding how S1/S2 compartments form. Because S1/S2s follow Xist-rich/-poor domains (Fig. 4f ${ }^{28}$, we investigated whether Xist is required. When Xist was deleted from the $\mathrm{Xi}$ after establishment of XCI in fibroblasts $\left(\mathrm{Xi}^{\Delta X i s t}\right)^{44}$, we observed failed SMCHD1 targeting to the Xi, consistent with Xist being required to recruit SMCHD1 (Fig. 5a, b, Supplementary Fig. 11$)^{27,28}$. We then performed in situ $\mathrm{Hi}-\mathrm{C}$ on $\mathrm{Xi}^{\Delta X i s t}$ fibroblasts. Deleting Xist on the Xi had no apparent effects on the Xa (Supplementary Fig. 2a), as expected. However, $\mathrm{Xi}^{\Delta X i s t}$ had a striking absence of S1/S2 compartments (Fig. 5c, top-right), instead showing homogeneous long-range interactions within each megadomain. The pronounced checkerboard pattern of the Smchd $1^{-1-} \mathrm{Xi}$ (Fig. 5c, bottom-middle) did not appear on the Pearson's correlation map of $\mathrm{Xi}^{\Delta X i s t}$ (Fig. $5 \mathrm{c}$, bottom-right). Moreover, PC1 captured only two megadomains for $\mathrm{Xi}^{\Delta X i s t}$ (Fig. 5d, bottom tracks), rather than the $\sim 25 \mathrm{~S} 1 / \mathrm{S} 2 \mathrm{~s}$ observed for the Smchd $1^{-1-} \mathrm{Xi}$ (Fig. 5d, middle tracks). These data indicate that, despite a failure of SMCHD1 recruitment, depleting Xist RNA does not unveil S1/S2 compartments. Xist RNA is therefore required to form S1/S2 compartments on the $\mathrm{Xi}$.

PRC1 partitions the $\mathrm{Xi}$ into $\mathrm{S1} / \mathrm{S} 2$ compartments. The $\mathrm{Xi}$ specific S1/S2 compartments provided an excellent opportunity to explore the idea that compartments arise from co-segregation of chromatin with similar epigenetic states-possibly through a biophysical process known as "LLPS"10,11,45. We postulated that Xist RNA drives $\mathrm{Xi}$ compartmentalization by concentrating chromatin factors with self-associating properties. Moreover, such factors must be preferentially bound to Xist-rich S1 domains. One such candidate is PRC1. PRC1 catalyzes H2AK119 ubiquitylation (H2AK119ub), compacts chromatin, and is known to be enriched on the $\mathrm{Xi}$ in an Xist-dependent manner (Fig. 5f, Supplementary Fig. 11 $)^{46-51}$. Furthermore, PRC1 has the potential to polymerize ${ }^{52}$ and promote small self-interacting domains at its target loci on autosomes $46,51,53,54$.

Given these observations, we hypothesized that PRC1 action may underlie S1/S2 segregation. Indeed, in Smchd1 $1^{-/}$cells, H2AK119ub remained enriched on the Xi (Fig. 5g), indicating that PRC1 recruitment can still occur without SMCHD1. Allelespecific ChIP-seq revealed that H2AK119ub patterns mirrored the crest-and-trough pattern of Xist binding (WT, $r=0.84$; Smchd $\left.1^{-/-}, r=0.95\right)$, correlating strongly with alternating S1/S2 profiles $(r=0.86)$ (Fig. 6a). As was the case for Xist and H3K27me3 densities, however, H2AK119ub density was heightened in the S1 compartment (Fig. 6a, b) - again consistent with the idea that Xist and its ensemble of recruited proteins being trapped in S1. Thus, Xist preferentially enriches PRC1 in the S1 domains in both WT and Smchd1 $1^{-/}$backgrounds.

We next asked if depleting PRC1 affects S1/S2 compartments in Smchd1 $1^{-/}$MEFs, where S1/S2s reemerged (Fig. 1). We 
a

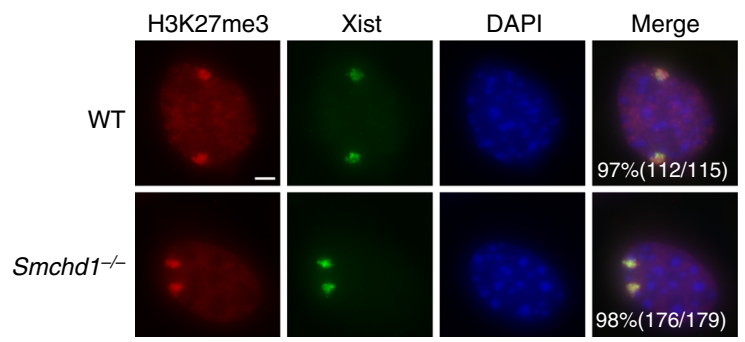

C

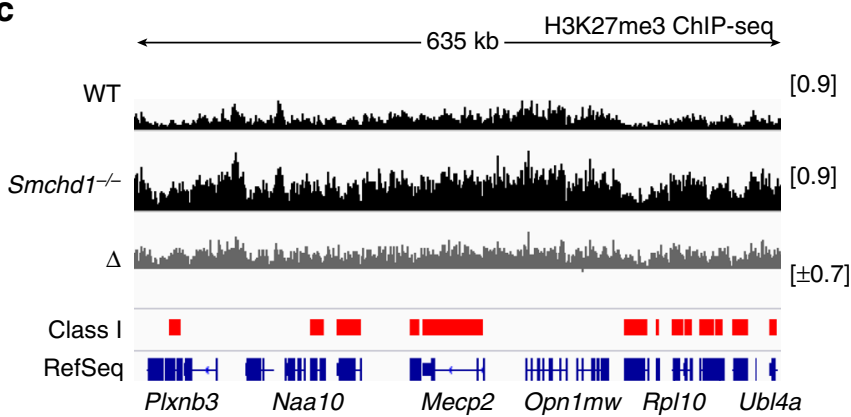

b

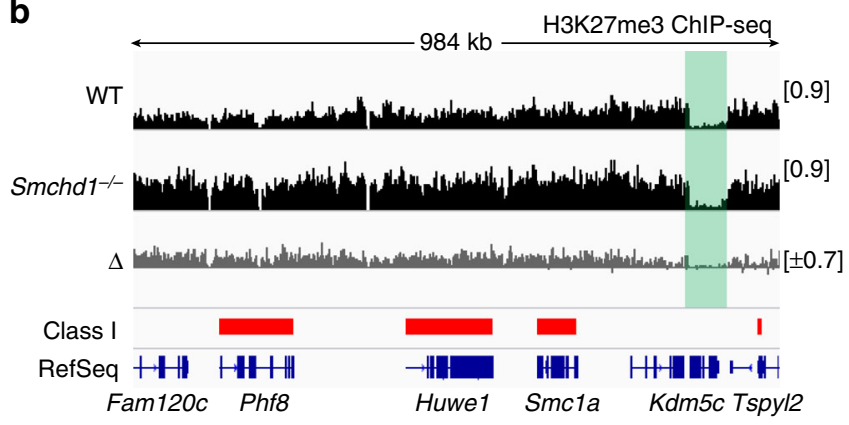

d

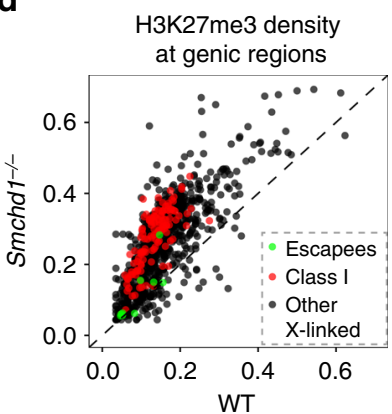

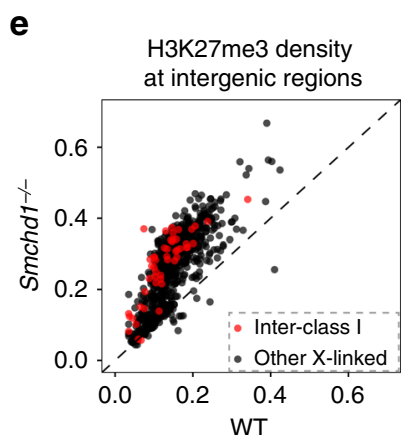

f

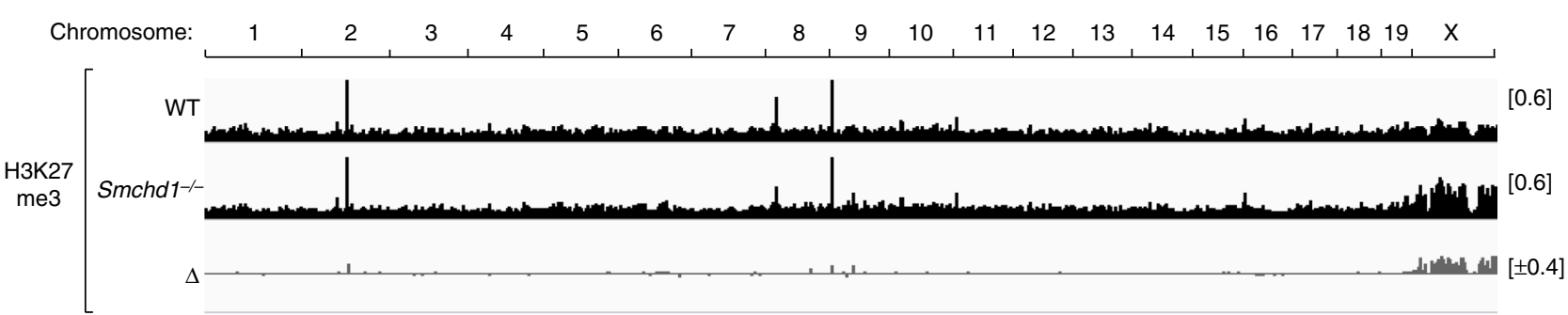

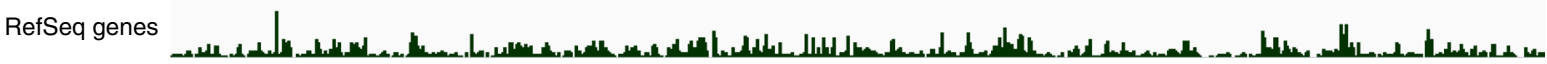

g

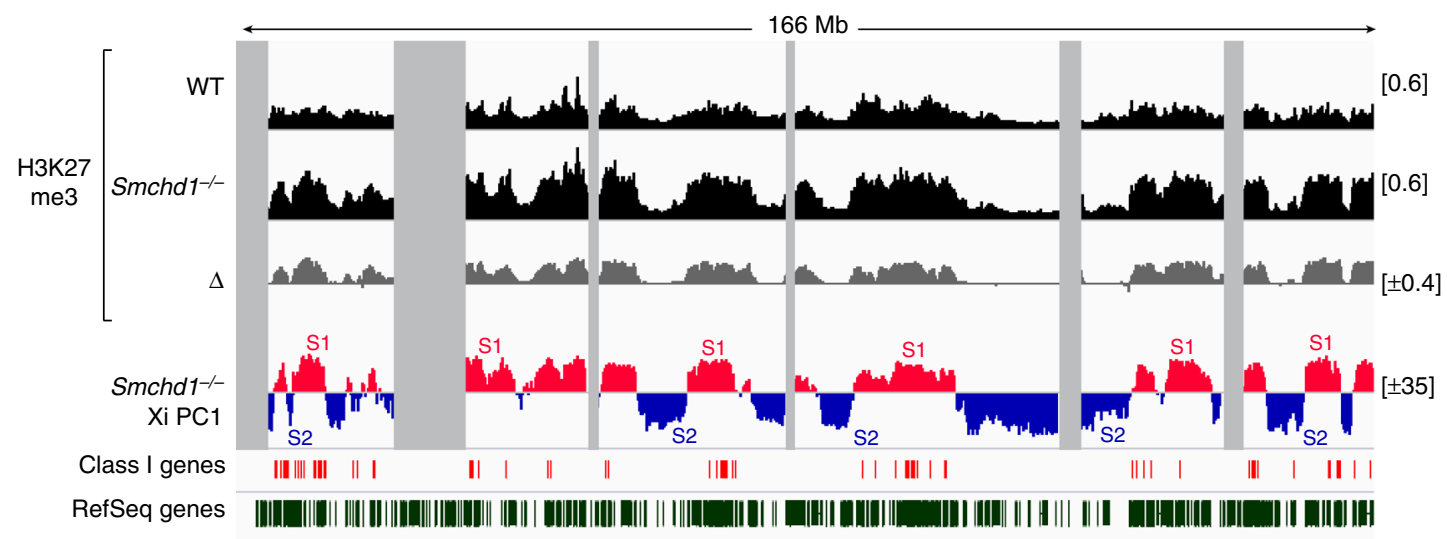

h

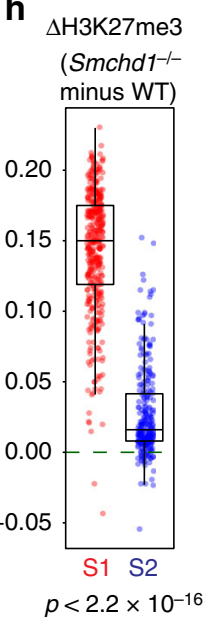

knocked down the catalytic subunits of PRC1-RING1A and RING1B ("PRC1 depletion") —and observed significantly attenuated H2AK119ub signals by western blot and immunofluorescence (Supplementary Fig. 12). In situ Hi-C revealed that, whereas the $\mathrm{Xa}$ showed no obvious changes (Supplementary Fig. 13), significant blunting of S1/S2 compartments was observed on the PRC1-depleted Xi (Fig. 6c). Contact heatmaps revealed that long-range interactions within each megadomain became more homogeneous (Fig. 6c, top), with the checkerboard pattern on the Pearson's correlation map markedly weakened (Fig. 6c, bottom). In support, PC1 captured two megadomains on the $\mathrm{Xi}$, but not compromised S1/S2s (Fig. 6d). Together, we conclude that PRC1 partitions the $\mathrm{Xi}$ into S1/S2 compartments.

To strengthen our conclusion, we also knocked down HNRNPK, a protein required for recruiting PRC1 to the $\mathrm{Xi}^{24,55,56}$. HNRNPK depletion recapitulates PRC1 depletion, causing a weakened checkerboard pattern on the Smchd1 ${ }^{-/}$Xi (Fig. 6c, right). Consistently, PC1 captured megadomains instead 
Fig. 3 Aberrant accumulation of H3K27me3 in the S1 compartment on the Smchd1-/- inactive X chromosome (Xi). a Immuno-RNA-fluorescent in situ hybridization (immuno-RNA-FISH) for H3K27me3 and Xist on wild-type (WT) and Smchd1 ${ }^{-/-}$mouse embryonic fibroblasts (MEFs). Number of cells with H3K27me3 foci co-localizing with Xist clouds is shown. Scale bar, $5 \mu \mathrm{m}$. b H3K27me3 profiles for a representative X-linked region harboring several Class I genes and an escapee $(K d m 5 c)$. Red bars, Class I genes. Scales shown in brackets. $\Delta$, Smchd1-/- minus WT. Note that we displayed the "comp" tracks (compiled from all reads) of H3K27me3 ChIP-seq (chromatin immunoprecipitation followed by deep sequencing), as most of the H3K27me3 signals are from the Xi. Allele-specific tracks have been deposited to Gene Expression Omnibus (GEO) (GSE116413). c H3K27me3 profiles for another representative $X$-linked region. $\mathbf{d}$ H3K27me3 density in gene bodies between WT ( $x$ axis) vs. Smchd1-/- ( $y$ axis) MEFs. Three categories of X-linked genes are shown. e H3K27me3 density in X-linked intergenic regions between WT ( $x$ axis) vs. Smchd1-/- ( $y$ axis) MEFs. Inter-Class I, intergenic regions flanked by two Class I genes. $\mathbf{f ~ H 3 K 2 7 m e 3 ~ e n r i c h m e n t ~ p r o f i l e s ~ a c r o s s ~ t h e ~ e n t i r e ~ g e n o m e . ~} \mathbf{g}$ H3K27me3 enrichment profiles across the $\mathrm{X}$ chromosome. Gray areas, unmappable regions. Also shown are the locations of Class I genes (red bars), and S1/S2 compartments in Smchd1-/- MEFs. $\mathbf{h}$ Box plots comparing the difference in H3K27me3 density between WT and Smchd1 ${ }^{-/-}$cells of each 200-kb bin in S1 vs. S2 compartments. $P$ values are given by the Wilcoxon's rank-sum test (unpaired, one-sided). Midline, median. Top and bottom of the box, first and third quartile. Whiskers, extension from the top or bottom to the furthest datum within 1.5 times the interquartile range

of attenuated S1/S2 compartments (Fig. 6d). This result supports a role for PRC1 in driving S1/S2 formation. Notably, a fainter checkerboard pattern remained visible on the HNRNPK-depleted $\mathrm{Xi}$, and to a lesser degree, on the PRC1-depleted Xi (Fig. 6c, bottom). This residual S1/S2-like structure was too weak to be captured by PC1. However, PC2 exhibited an alternating profile reminiscent of S1/S2s (Fig. 6d). Together, these data suggest that depleting HNRNPK or PRC1 significantly compromised, but did not completely eradicate, the S1/S2 structure, likely because of incomplete knockdown (KD) of these factors.

PRC1 partially obscures megadomains. Interestingly, despite lacking two key structural factors, SMCHD1 and PRC1, insulation between the two Xi-specific megadomains became paradoxically strengthened. Notably, megadomain contours became sharper (compare Fig. 6c, WT vs. PRC1/HNRNPK KD), coinciding with reduced inter-megadomain contacts in PRC1/ HNRNPK-depleted cells (Fig. 6e). By contrast, ablating Smchd1 alone lacked this phenotype, suggesting an effect unique to depleting PRC1. Given similarly strengthened megadomains when either Xist or PRC1 was depleted (Fig. $5 \mathrm{c}-\mathrm{e}$ ), we suggest that Xist facilitates long-range interactions across the Dxz4 boundary through self-association between PRC1-enriched chromatin, which thereby partially obscures megadomains. Loss of either Xist, HNRNPK, or PRC1 would therefore result in sharper megadomains. We conclude that PRC1 mediates longrange interactions that define the overall contours of the Xi superstructure.

To investigate how Xist RNA recruits SMCHD1, we screened the Xist interactome $e^{21,55,57}$ and found that HNRNPK depletion abolished SMCHD1 recruitment (Fig. 7a, Supplementary Fig. 14b). Given that HNRNPK is also required for targeting PRC1 to the $\mathrm{Xi}^{24,55,56}$, we examined the relationship of PRC1 to SMCHD1 localization. Strikingly, PRC1-depleted MEFs also failed to recruit SMCHD1 (Fig. 7a, Supplementary Fig. 14b). To determine if the PRC1 mark, H2AK119ub, is required for SMCHD1 recruitment, we treated cells with MG132, a proteasome inhibitor that depletes H2AK119ub by blocking the recycling of free ubiquitin (Supplementary Fig. $14 \mathrm{c}, \mathrm{d})^{58}$. Intriguingly, MG132 treatment also abolished SMCHD1 localization (Fig. 7b). Thus, it is likely that, beyond PRC1, the H2AK119ub mark itself is essential for SMCHD1 recruitment. In contrast, depleting EED, an essential PRC2 subunit, did not affect SMCHD1 targeting (Fig. 7a, Supplementary Fig. 14b), consistent with a human study ${ }^{27}$. Knocking down other Xistinteracting proteins-such as SPEN (Spen family transcriptional repressor), RBM15 (RNA-binding motif protein 15), or LBR (lamin B receptor)-did not affect SMCHD1 recruitment (Supplementary Fig. 14a, b). These data indicate that the effect of depleting HNRNPK, PRC1, and H2AK119ub on SMCHD1 localization is specific. Xist RNA therefore recruits SMCHD1 via a mechanism dependent on HNRNPK, PRC1, and H2AK119ub.

To examine the general relationship between PRC1 and SMCHD1, we examined their distribution on autosomes. As expected, the H3K27me3 mark was enriched near H2K119ub peaks on autosomes (Fig. 7c, Supplementary Fig. 14e). By contrast, SMCHD1 was not enriched at these autosomal Polycomb targets. We conclude that PRC1 and H2AK119ub may not be sufficient for SMCHD1 recruitment at autosomal sites. Thus, autosomal and $\mathrm{Xi}$ environments may be distinct.

Given a strong effect of the PRC1 pathway on SMCHD1 recruitment, we asked if its perturbation affects the merging of S1/S2 compartments, a key function of SMCHD128. We performed in situ Hi-C on HNRNPK- and PRC1-KD cells in otherwise WT cells. Following PRC1 KD, S1/S2s did not become visible (Fig. 7d, e). Depleting HNRNPK also did not unveil S1/S2s (Fig. 7d, e). Rather, both conditions caused significantly sharpened megadomains and decreased inter-megadomain interactions (Fig. 7d-f). This finding is in line with a role for PRC1 in promoting long-range interactions that span the Dxz4 boundary. The lack of re-emergent S1/S2 compartments despite a failure of SMCHD1 recruitment (Fig. 7a) is also consistent with PRC1 being necessary to form S1/S2s.

Collectively, these data demonstrate that Xist recruits PRC1 to partition the $\mathrm{Xi}$ into $\mathrm{S} 1 / \mathrm{S} 2$ compartments (Figs. 5-7), and SMCHD1 is required to merge S1/S2s into the compartment-less $\mathrm{Xi}$, even during $\mathrm{Xi}$ maintenance (Fig. 1). Moreover, the requirement of PRC1 in SMCHD1 recruitment necessitates S1/ S2 compartments being formed prior to SMCHD1 localization, providing a potential mechanism that coordinates the stepwise folding process.

\section{Discussion}

Here we have provided insight into the stepwise folding mechanism of the Xi (Fig. 8a), a process we previously likened to "origami" 28 . Similar to origami, the folding and unfolding of the Xi appear to follow the "principle of reversibility" (unfolding taking the reverse pathway of folding). During de novo XCI, A/B compartments are first remodeled into S1/S2 structures. This step depends on Xist RNA (Fig. 5) and occurs as Xist spreads across the chromosome 28 . One of the factors recruited by Xist is PRC1 (Fig. 5f ${ }^{21,47-50,55}$. We propose that, once recruited by Xist, the uneven distribution of PRC1 on the Xi aids in its phase separation into S1/S2 compartments, with Xist- and H2AK119-enriched chromatin partitioning into the S1 compartment (Figs. 6, 8b). Once PRC1 successfully re-compartmentalizes the $\mathrm{Xi}$ into the S1/ S2 transitional state, SMCHD1 is recruited to merge S1/S2s to create a super-structure-the "one compartment" state that gives the appearance of being "compartment-less." In light of the complementary view of genome architecture offered by ligation- 
a

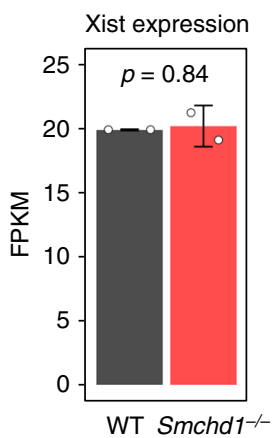

b $639 \mathrm{~kb}$

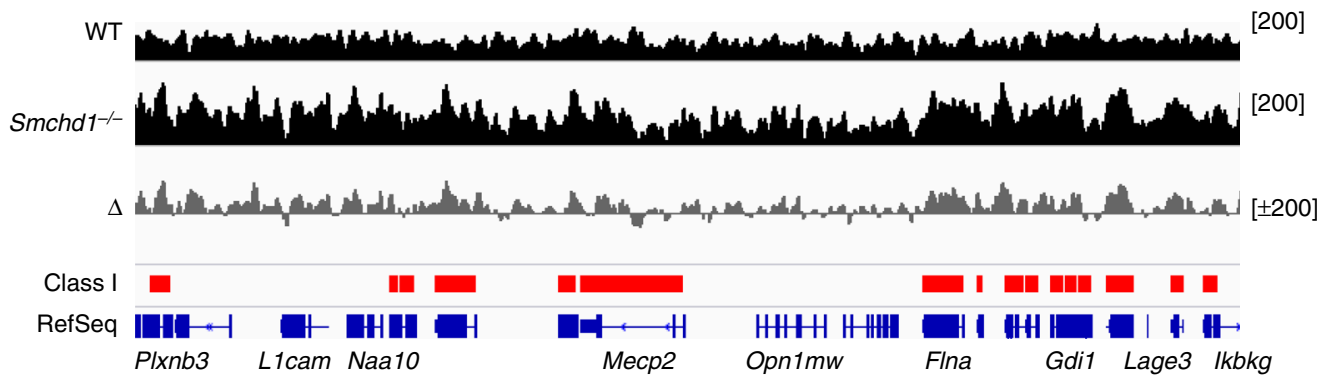

d

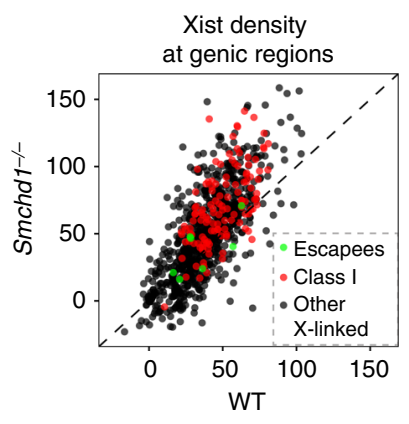

e

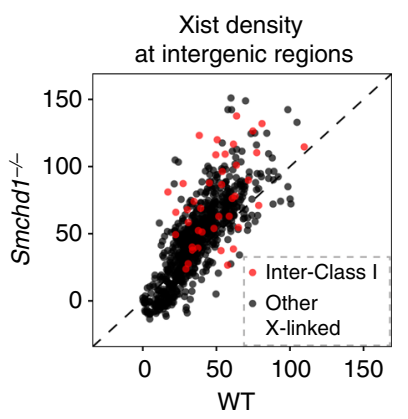

f

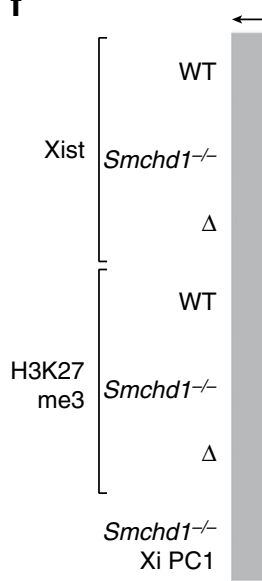

Class I genes

RefSeq genes
$991 \mathrm{~kb}$

Xist CHART-seq

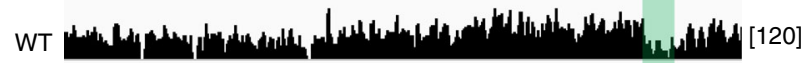

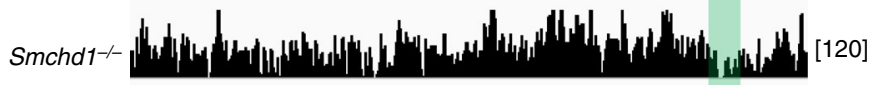

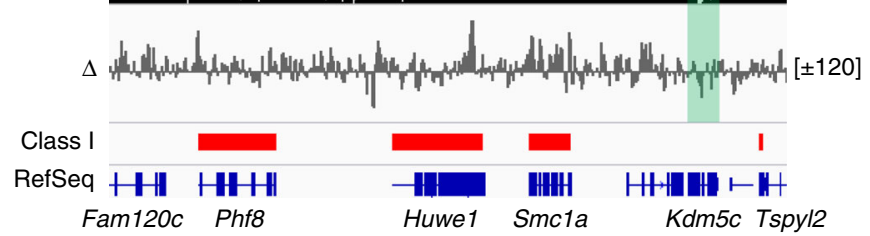

C

Xist CHART-seq

g

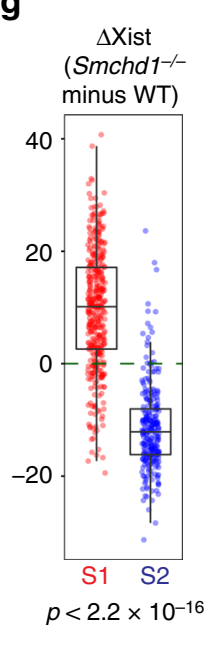

Fig. 4 Ablating Smchd1 traps Xist RNA in the S1 compartment. a RNA-sequencing (RNA-seq) fragments per kilobase of transcript per million mapped reads (FPKM) values of Xist in wild-type (WT) and Smchd1 ${ }^{-/-}$mouse embryonic fibroblasts (MEFs). RNA-seq data from two WT and two Smchd1-/- clones treated with dimethyl sulfoxide (DMSO) were analyzed. $P$ values are given by $t$ test (unpaired, two-sided). Error bars, s.d. b Xist CHART (capture hybridization analysis of RNA target) profiles for a representative X-linked region harboring several Class I genes. Red bars, Class I genes. Scales shown in brackets. $\Delta$, Smchd1 ${ }^{-/-}$minus WT. c Xist CHART profiles for another representative X-linked region. Green-shaded area, an escapee (Kdm5c). d Xist density in gene bodies between WT ( $x$ axis) vs. Smchd1-/- ( $y$ axis) MEFs. Three categories of X-linked genes are shown. e Xist density in X-linked intergenic regions between WT ( $x$ axis) vs. Smchd1 ${ }^{-1-}$ ( $y$ axis) cells. Inter-Class I, intergenic regions flanked by two Class I genes. $\mathbf{f}$ Xist enrichment profiles across the $X$ chromosome. Gray areas, unmappable regions. Also shown are H3K27me3 ChIP (chromatin immunoprecipitation followed by deep sequencing) profiles, the locations of Class I genes (red bars), and S1/S2 compartments in Smchd1-/- MEFs. $\mathbf{g}$ Box plots comparing the difference in Xist density between WT and Smchd1 ${ }^{-/}$- cells of each 200-kb bin in S1 vs. S2 compartments. $P$ values are given by the Wilcoxon's rank-sum test (unpaired, one-sided). Midline, median. Top and bottom of the box, first and third quartile. Whiskers, extension from the top or bottom to the furthest datum within 1.5 times the interquartile range

independent methods ${ }^{59,60}$, we suggest that the Xi might not be completely compartment-less, as it might harbor an underlying organization not effectively captured by Hi-C.

Central to the stepwise folding mechanism is a requirement for PRC1 to either directly or indirectly enable SMCHD1 recruitment
(Fig. 7), as also described in a recent work ${ }^{41}$. Here our perturbation studies reveal that architectural regulation is a key aspect of the relationship between PRC1 and SMCHD1. In the post-XCI state, ablating key components of the Xist-HNRNPK-PRC1SMCHD1 pathway unravels the architectural layers of the $\mathrm{Xi}$, one 
a

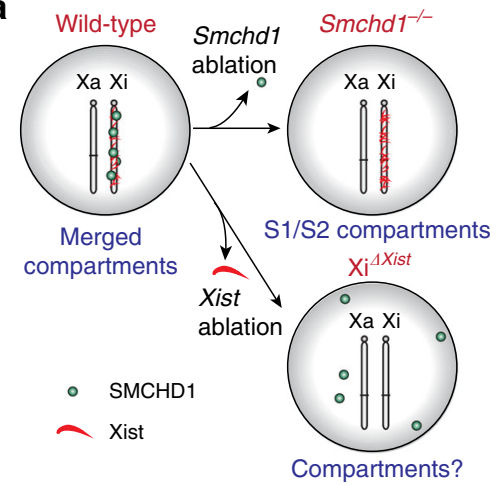

b

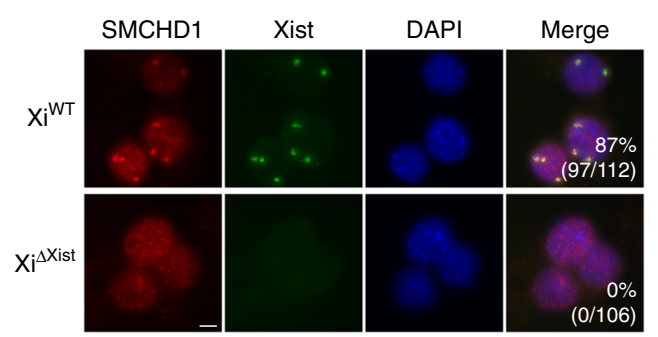

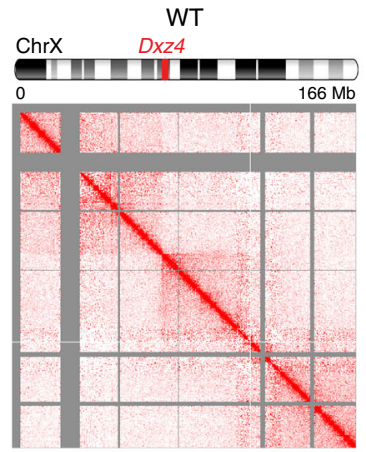

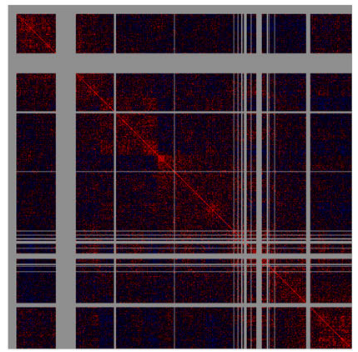

\section{ChrX}

Smchd1 $1^{-1}$ Dxz4
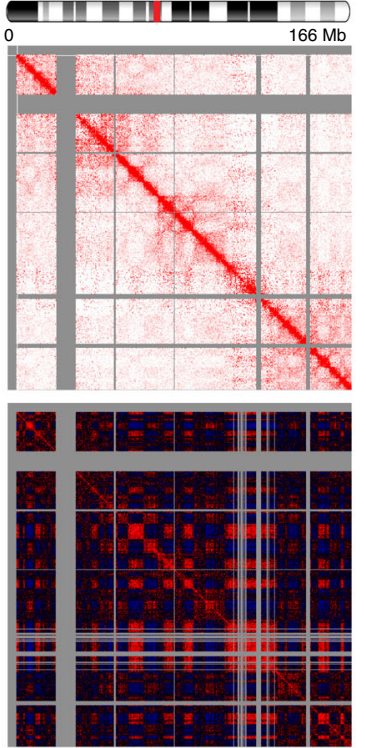

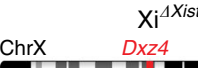
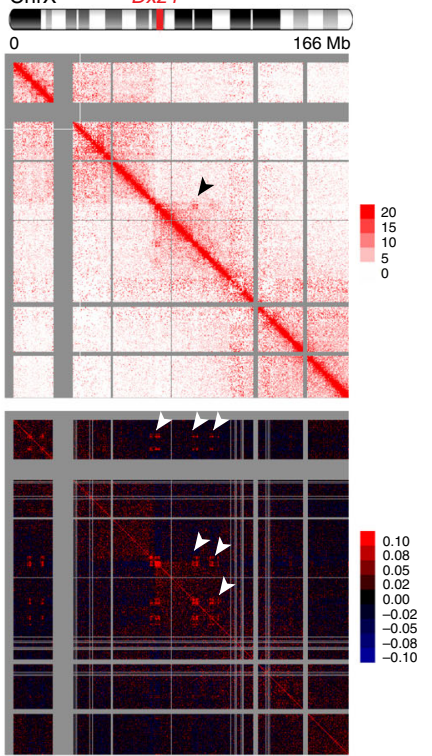

Xist
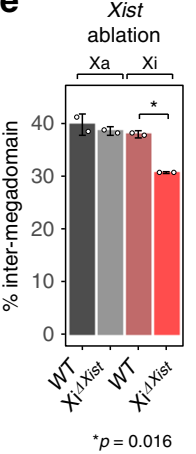

d
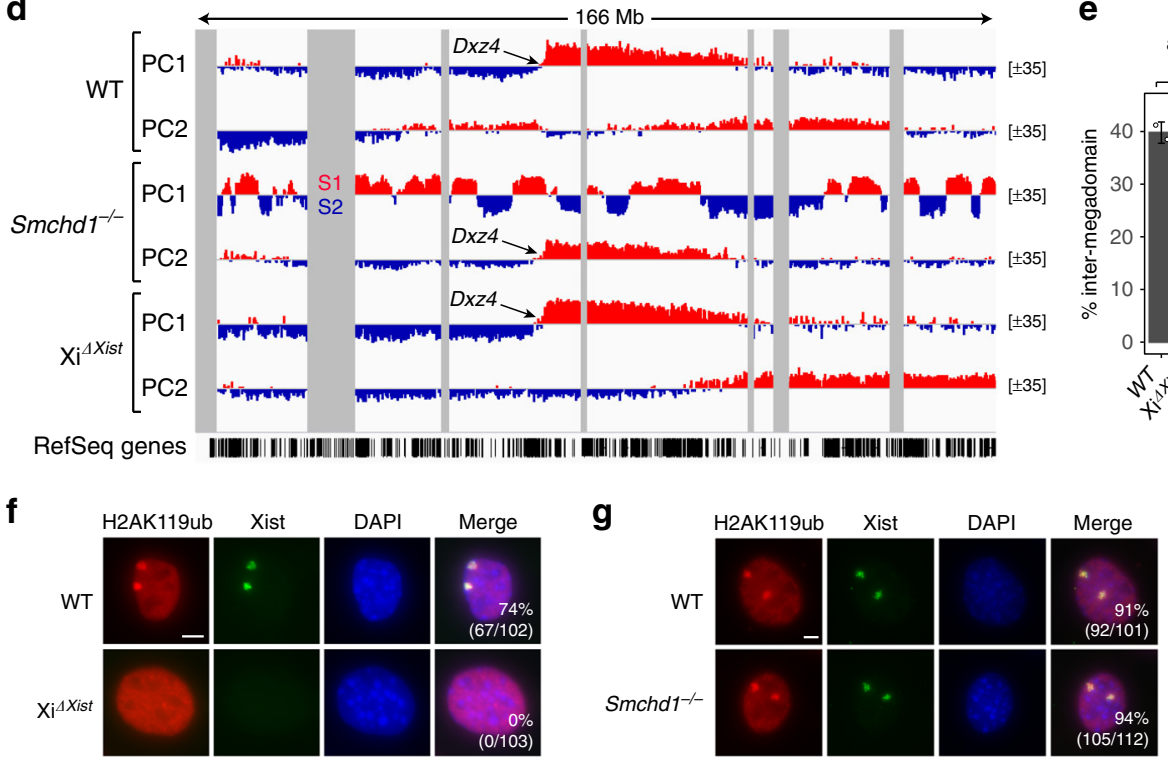

at a time, enabling the re-emergence of various hidden structures -in the reverse order of appearance during de novo XCI. Indeed, the transitional S1/S2 compartments reappear upon Smchd1 ablation. Depleting PRC1 or HNRNPK in a SMCHD1-deficient background does not resurrect A/B compartments (Fig. 6), possibly because factors associated with active transcription may be necessary to segregate A from B compartments ${ }^{61}$. Instead, we observed a disorganized and de-compartmentalized Xi, where S1/ S2s are significantly weakened. In the wild-type background, depleting Xist, HNRNPK, or PRC1 also decompartmentalizes the Xi (Figs. 5, 7), although S1/S2 compartments may be resurrected transiently during this process. Interestingly, the aberrant $\mathrm{Xi}$ in 
Fig. 5 Ablating Xist RNA does not reveal S1/S2 compartments despite failed structural maintenance of chromosomes hinge domain containing 1 (SMCHD1) recruitment. a Schematic representation of the epigenetic status of the inactive $\mathrm{X}$ chromosome (Xi) in wild-type (WT), Smchd7 ${ }^{-/-}$, and $\mathrm{Xi}^{\Delta \mathrm{Xist}}$ fibroblasts. b Immuno-RNA-fluorescent in situ hybridization (Immuno-RNA-FISH) for SMCHD1 and Xist on WT [XiWT, 2lox (Xist+)] and XisXist fibroblasts. Scale bar, $10 \mu \mathrm{m}$. c Depth-corrected chromatin interaction maps of the Xi in WT, Smchd1-/-, and XiAXist fibroblasts binned at 200-kb resolution (top) and the corresponding Pearson's correlation maps (bottom). Gray-shaded areas, unmappable regions. Also see Supplementary Fig. 2a for active X chromosome (Xa) maps. Note that in the Xi maps of Xi山Xist fibroblasts, the "super-loops" (arrowheads) formed by association between Xi regions exhibiting ATAC-seq (assay for transposase-accessible chromatin using sequencing) accessibility, BRG1 binding, cohesin binding, and topologically associated domain (TAD)like structures not seen on the WT Xi can also be observed. Please see our reanalysis ${ }^{68}$ of $\mathrm{Hi}-\mathrm{C}$ data from the same $\mathrm{Xi}^{\Delta \mathrm{Xist}}$ fibroblasts ${ }^{21}$ for in-depth description. d Principal component 1 (PC1) and PC2 values of the Xi. Gray-shaded areas, unmappable regions. Also see Supplementary Fig. 2b for PCs of the Xa. e Bar plots displaying the fraction of long-range interactions ( $>10 \mathrm{Mb}$ ) that span the megadomain boundary ("inter-megadomain" interactions) on the $\mathrm{Xa}$ and $\mathrm{Xi}$ in $\mathrm{WT}$ and $\mathrm{Xi}_{\mathrm{i}}^{\Delta X_{i s t}}$ fibroblasts. Two replicates were analyzed, with $P$-values determined by the $t$ test (unpaired, one-sided). Error bars, s.d. f Immuno-RNA-FISH for H2AK119ub and Xist on WT [2lox(Xist+)] and XidXist fibroblasts. Number of cells with Xist clouds and co-localizing H2AK119ub foci is shown. Scale bar, $5 \mu \mathrm{m}$. $\mathbf{g}$ Immuno-RNA-FISH for H2AK119ub and Xist on WT and Smchd1-/- MEFs. Number of cells with H2AK119ub foci colocalizing with Xist clouds is shown. Scale bar, $5 \mu \mathrm{m}$

these cells exhibits stronger insulation between the two megadomains. We speculate that PRC1's known self-associating and/ or chromatin-compacting properties not only facilitate shortrange interactions through condensation but also long-range interactions across the Dxz4 border (Fig. 8b), explaining why its loss sharpens the megadomain boundary. Consistently, deleting Repeat $\mathrm{B}$, the RNA motif required for Xist to interact with HNRNPK and to recruit PRC1, also causes a disorganized $\mathrm{Xi}$ and reduces inter-megadomain interaction ${ }^{24}$. In summary, the antagonistic role of SMCHD1 on PRC1-mediated Xi compartments and the obscuring effect of PRC1 on megadomains illustrate that the $\mathrm{Xi}$ is shaped by multiple, sometimes competing folding mechanisms (Fig. 8c). Thus, the $\mathrm{Xi}$ is far from the "structure-less" body that it was once thought to be.

A role for PRC1 in S1/S2 compartmentalization supports a mechanism involving LLPS, an idea widely proposed to explain chromosome compartmentalization. A major gap has been the lack of evidence for critical chromatin factors with known phaseseparating property. In this regard, a role for PRC1 is especially attractive, given recent evidence that PRC1 forms liquid droplets in vitro and nuclear condensates in vivo that are sensitive to hexanediol $^{12,13}$. Our data indicating that depleting PRC1 abolishes large-scale S1/S2 compartments lends direct support to the hypothesis that LLPS contributes to chromosome compartmentalization.

Our work also demonstrates a clear functional relevance for $\mathrm{S} 1 /$ S2 compartments. Indeed, the S1/S2 organization is important for both global and regional Xist spreading, and associated gene silencing. During de novo XCI, failure to merge S1/S2s compromises regional Xist spreading, resulting in eroded $\mathrm{H} 3 \mathrm{~K} 27 \mathrm{me} 3$ domains and impaired gene silencing, specifically at regions harboring SMCHD1-sensitive "Class I genes"28. By contrast, in post-XCI cells, depleting SMCHD1 did not affect regional spreading, suggesting that once Xist establishes an epigenetic memory in Class I regions, SMCHD1 becomes dispensable for local Xist spreading. An Xist-induced epigenetic memory is in line with earlier observations ${ }^{43,50,62}$. While regional Xist spreading is preserved in post-XCI cells, ablating Smchd1 affects global Xist distribution. Spatial segregation between S1 and S2 traps Xist RNA in the S1 compartment where it is produced, rendering the RNA unable to spread as efficiently into adjoining S2 structures. As a consequence, $\mathrm{H} 3 \mathrm{~K} 27 \mathrm{me} 3$ marks accumulate in S1 domains (Fig. 3g, h). H2AK119ub marks also accumulate in S1 (Fig. 6a, b).

In addition to merging S1/S2 compartments, SMCHD1 also attenuates Xi TADs. Ablating Smchd1 strengthens Xi TADs both during de novo $\mathrm{XCI}^{28}$ and in the maintenance phase (Supplementary Fig. 4). Notably, TADs on the $S m c h d 1^{-/-} \mathrm{Xi}$ remain weaker than those on the $\mathrm{Xa}$, indicating the existence of SMCHD1-independent mechanisms. Inefficient cohesin loading and sliding due to attenuated transcription ${ }^{63}$, impaired CTCF binding by DNA methylation ${ }^{64}$, and other architectural proteins at play on the $\mathrm{Xi}$ are candidate mechanisms to be explored in the future.

Altogether, perturbing SMCHD1 and S1/S2 structures result in a weakened silent state that is more easily disrupted by pharmacological DNA demethylation (Fig. 2). Our findings support a synergy between SMCHD1 and DNA methylation contributes to $\mathrm{Xi}$ maintenance. The heightened reactivation efficiency in Smchd $1^{-1}$ cells seems at odds with elevated repressive histone marks on the Xi (Figs. 3, 6). As reawakening the healthy allele of disease genes on the $\mathrm{Xi}$ presents a therapeutic opportunity in $\mathrm{X}$ linked disorders ${ }^{38}$, our finding suggests dual targeting of SMCHD1 and DNA methylation as a candidate approach in developing such treatment strategy.

Overall, our work complements several recent studies on the role of SMCHD1 in $\mathrm{XCI}^{28,35,36,41,65}$. There are, however, several notable differences. For instance, one study indicated that ablating Smchd1 in post-XCI cells caused a reversion to "A/B" compartments similar to those on the $\mathrm{Xa}^{36}$, rather than anveiling of the S1/S2 compartments we describe. There are several reasons to believe that the compartments described by Gdula et al. ${ }^{36}$ may be more akin to S1/S2s. First, they are larger than classical A/B compartments and are partitioned along the Xi like S1/S2s. Second, the partitioning follows an S1/S2 pattern that is distinguished by Xist, H3K27me3, and H2AK119ub densities, rather than the transcriptional states that typify $\mathrm{A} / \mathrm{B}$ compartments. Third, classical A/B compartments do not require Xist RNA, HNRNPK, and PRC1, but the structures we describe clearly do. Thus, ablating Smchd1 results in compartment structures (S1/S2) that have properties that distinguish them from classical $A / B$ compartments. A second notable difference regards the enrichment of H3K27me3 following SMCHD1 depletion. Another group has reported that ablating $S m c h d 1$ in post-XCI cells also causes an overall increase of $\mathrm{H} 3 \mathrm{~K} 27 \mathrm{me} 3$ on the $\mathrm{Xi}^{65}$. However, we further linked the preferential accumulation of H3K27me3 on the S1 domains to altered distribution of Xist RNA and Xi topology. Altogether, these works highlight an exciting area of $3 \mathrm{D}$ chromosome biology and underscores the utility of the $\mathrm{Xi}$ for dissecting structure-function relationships.

\section{Methods}

Cell lines. The clonal F1 hybrid MEF line (EY.T4), Xi ${ }^{\Delta X i s t}$ fibroblasts (clone III.20), 2lox(Xist+) fibroblasts (clone III.8), and a MEF line of pure Mus castaneous background have been described ${ }^{29,44,66}$. Cells were grown in MEF media [Dulbecco's modified Eagle's medium, high glucose, GlutaMAX supplement, pyruvate (10569044, Thermo Fisher Scientific), 10\% fetal bovine serum, $25 \mathrm{mM}$ HEPES, pH 7.2-7.5 (15630130, Thermo Fisher Scientific), 1× MEM non-essential amino acids (11140076, Thermo Fisher Scientific), 1× penicillin-streptomycin (15140163, Thermo Fisher Scientific), and $0.1 \mathrm{mM} \beta$-mercaptoethanol (21985023, Thermo 
a

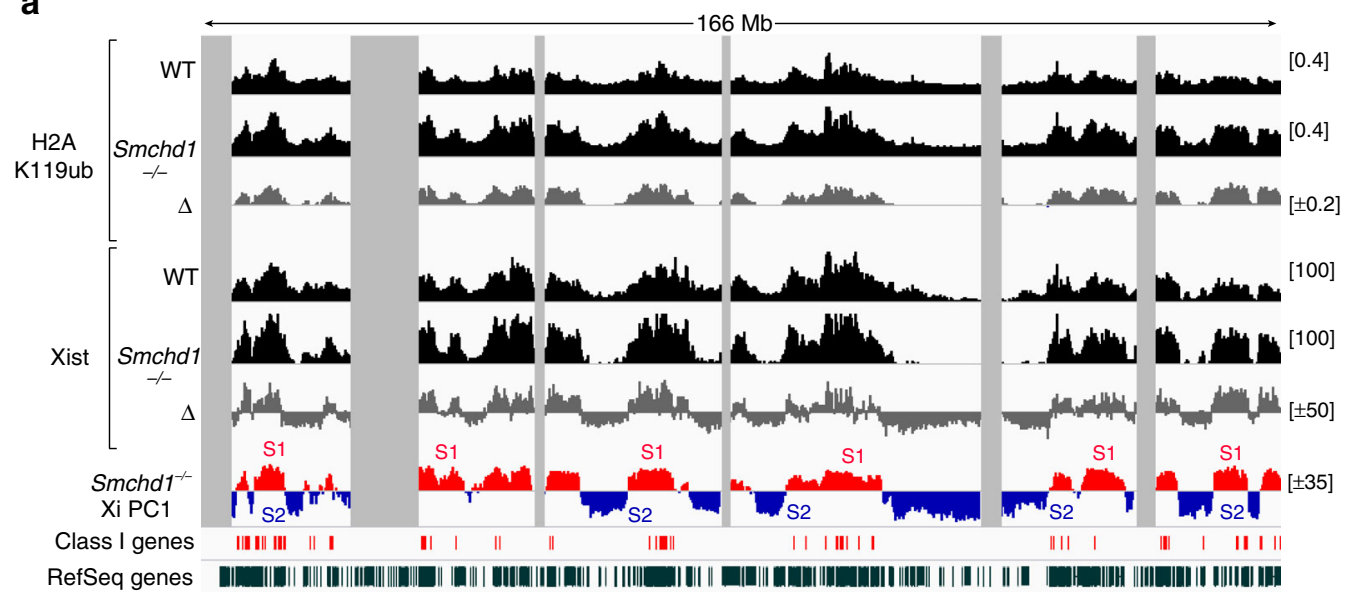

b

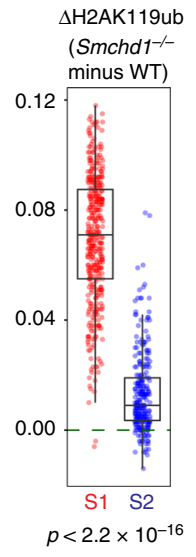

C

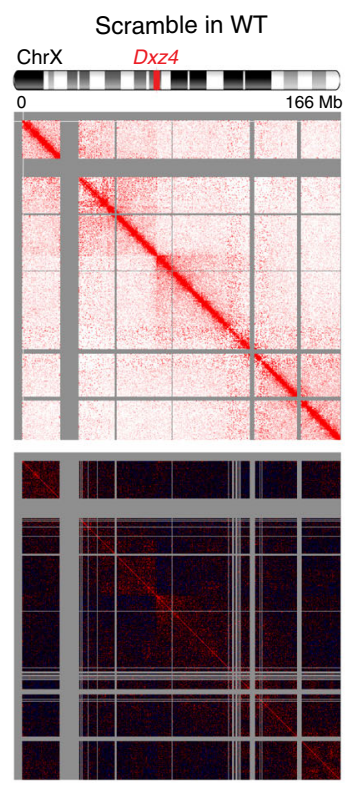

Scramble in Smchd1 ${ }^{-/}$ ChrX Dxz4 ChrX Dxz4
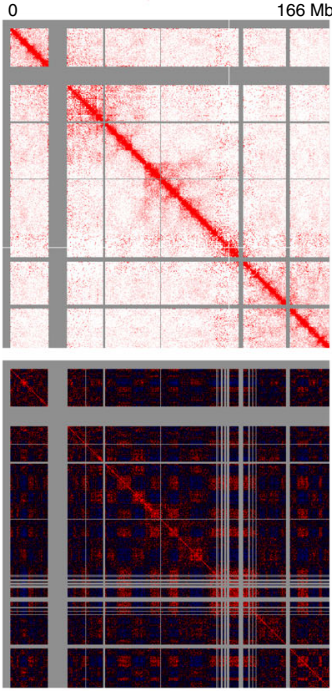

PRC1 KD in Smchd1 ${ }^{-/-}$ ChrX Dxz4
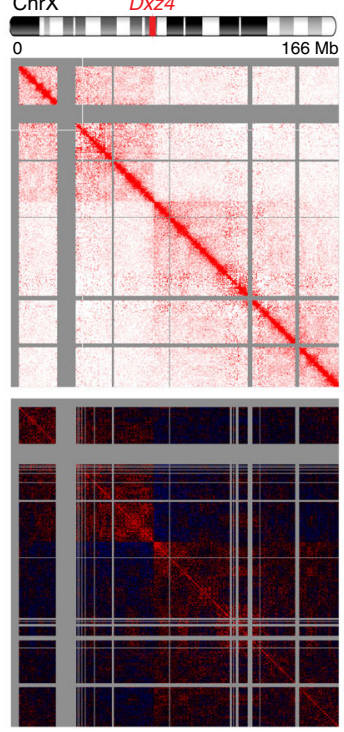

HNRNPK KD in Smchd1 $1^{-/}$
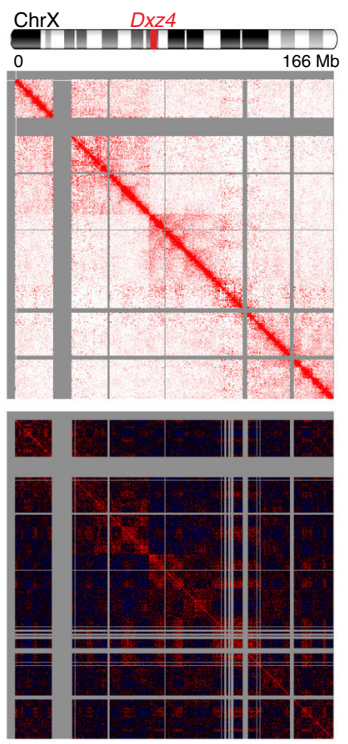

d

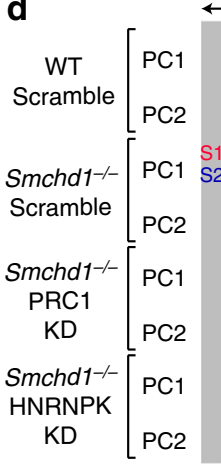

RefSeq genes

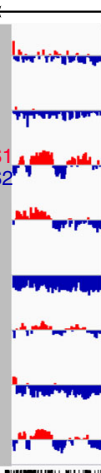

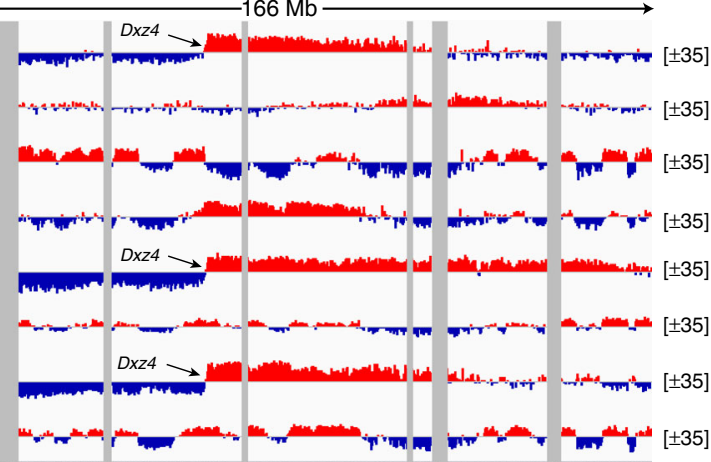

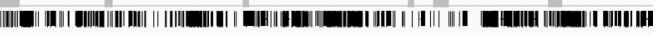

e

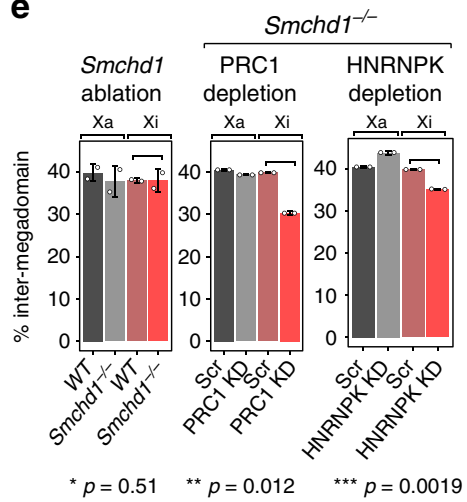

Fisher Scientific)]. Smchd1 ${ }^{-/-}$MEF lines were generated by transfecting the EY.T4 line using Lipofectamine LTX and Plus Reagent (15338100, Thermo Fisher Scientific) with plasmids (PX461; 48140, Addgene) expressing Cas9 D10A nickase, enhanced green fluorescent protein (EGFP), and a pair of guide RNAs targeting the first exon of $S m c h d 1$, as described previously ${ }^{28}$. To control off-target effects, two different pairs of guide RNAs were used (pair 1: GGGAGAGATGGCGCCGT CGA, CGAGAGGCCGGCGGGATCGC; pair 2: CTTGTTTGACCGGCGCGGGA, CACCGTCCTACAGCCGTCGA) (see Supplementary Data 3 for oligos used for cloning). WT control MEF lines were generated by transfecting EY.T4 with the PX461 plasmid without guide RNAs. At $24 \mathrm{~h}$ after transfection, cells were dissociated and transfected cells were sorted by EGFP into 96-well plates, with one cell plated in each well. MEF clones were screened by western blot using an antibody against SMCHD1 (Sigma, HPA039441). To confirm the presence of frame-shifting mutations, the CRISPR-targeted region was PCR amplified from genomic DNA (primers are listed in Supplementary Data 3 ) and subsequently cloned into a TOPO-TA vector (450030, Thermo Fisher Scientific) for Sanger sequencing. For RNA-seq, two WT and two Smchd1 $1^{-1-}$ clones were used. For in situ Hi-C, ChIPseq, and CHART-seq, WT clone 1 and Smchd1 $1^{-/-}$clone 1 were used.

For Aza (A3656, Sigma) treatment, we incubated WT and Smchd1 ${ }^{-/-}$MEFs with media containing either DMSO or Aza $(0.3 \mu \mathrm{M})$ for 5 days. Media supplemented with DMSO or Aza were changed daily.

For MG132 (M7449, Sigma) treatment, 0.1 million WT MEFs were plated in one well of a 12 -well plate (or 0.5 million in one well of a 6 -well plate). The next day, media were replaced with fresh media containing $10 \mu \mathrm{M}$ MG132. After 
Fig. 6 Depleting polycomb repressive complex 1 (PRC1) or heterogeneous nuclear ribonucleoprotein K (HNRNPK) disrupts S1/S2 compartments in Smchd1 ${ }^{-/-}$mouse embryonic fibroblasts (MEFs). a H2AK119ub enrichment profiles across the X chromosome. Gray areas, unmappable regions. Also shown are Xist CHART (capture hybridization analysis of RNA target) profiles, the locations of Class I genes (red bars), and S1/S2 compartments inSmchd1 1 /- MEFs. $\Delta$, Smchd1 $-/-$ minus wild type (WT). Shown are "comp" tracks (compiled from all reads) of H2AK119ub ChIP-seq (chromatin immunoprecipitation followed by deep sequencing). Allele-specific tracks have been deposited to Gene Expression Omnibus (GEO) (GSE116413). b Box plots comparing the difference in H2AK119ub density between WT and Smchd1-/- cells of each 200-kb bin in S1 vs. S2 compartments. $P$ values are given by the Wilcoxon's rank-sum test (unpaired, one-sided). Midline, median. Top and bottom of the box, first and third quartile. Whiskers, extension from the top or bottom to the furthest datum within 1.5 times the interquartile range. $\mathbf{c}$ Depth-corrected chromatin interaction maps of the inactive $X$ chromosome $(\mathrm{Xi})$ in WT MEFs treated with control (Scramble) small interfering RNA (siRNA), and Smchd1-/- MEFs treated with control, RING1A/RING1B (PRC1 KD), or HNRNPK (HNRNPK KD) siRNA binned at 200-kb resolution (top) and the corresponding Pearson's correlation maps (bottom). Gray-shaded areas, unmappable regions. Also see Supplementary Fig. 13a for the active $X$ chromosome (Xa) maps. d Principal component 1 (PC1) and PC2 values of the Xi. Regions with positive PC1 values represent the S1 compartment or the telomeric megadomain. Gray-shaded areas, unmappable regions. Also see Supplementary Fig. 13b for PCs of the Xa. e Bar plots displaying the fraction of long-range interactions (>10 Mb) that span the Dxz4 megadomain boundary ("inter-megadomain" interactions) under different conditions. Two replicates were analyzed, with $P$ values determined by the $t$ test (unpaired, one-sided). Error bars, s.d.

incubation for $6 \mathrm{~h}$, cells were harvested for immuno-RNA-fluorescent in situ hybridization (immuno-RNA-FISH) and western blot analyses.

RNA-FISH and immunostaining. Fibroblasts were grown overnight on glass coverslips coated with $0.2 \%$ gelatin. The next day, cells were rinsed in chilled phosphate-buffered saline (PBS) for $5 \mathrm{~min}$ three times, pre-extracted with CSKT buffer $(100 \mathrm{mM} \mathrm{NaCl}, 300 \mathrm{mM}$ sucrose, $10 \mathrm{mM}$ PIPES, $3 \mathrm{mM} \mathrm{MgCl}$, $0.5 \%$ Triton $\mathrm{X}-100, \mathrm{pH}$ 6.8) supplemented with $10 \mathrm{mM}$ ribonucleoside vanadyl complex (VRC; S1042S, NEB) for $10 \mathrm{~min}$ on ice, and fixed with $4 \%$ paraformaldehyde (15713, Electron Microscopy Sciences) in PBS at room temperature for $10 \mathrm{~min}$ (for $\mathrm{H} 2 \mathrm{AK} 119 \mathrm{ub}$ and $\mathrm{H} 3 \mathrm{~K} 27 \mathrm{me} 3$ ) or chilled $100 \%$ methanol at $-20^{\circ} \mathrm{C}$ for $10 \mathrm{~min}$ (for SMCHD1). After fixation, cells were washed with chilled PBS for 5 min, further permeabilized by CSKT with $10 \mathrm{mM}$ VRC for $10 \mathrm{~min}$ on ice, followed by three washes with chilled PBS for $5 \mathrm{~min}$. After blocking with 5\% normal goat serum (PCN5000, Thermo Fisher Scientific) with $0.6 \mathrm{U} / \mu$ l Protector RNase Inhibitor (3335402001, Sigma) in PBS at room temperature for $1 \mathrm{~h}$, immunostaining were performed using rabbit monoclonal H3K27me3 antibodies (GTX60892, GeneTex, 1:3000), rabbit monoclonal H2AK119ub antibodies (8240S, Cell Signaling, 1:1000), or rabbit polyclonal SMCHD1 antibodies (HPA039441, Sigma, 1:200) in antibody dilution buffer (5\% normal goat serum, $0.6 \mathrm{U} / \mu \mathrm{l}$ Protector RNase Inhibitor, and $0.2 \%$ Tween-20 in PBS) at room temperature for $1 \mathrm{~h}$. Following primary antibody incubation, cells were washed with PBST (PBS with $0.2 \%$ Tween-20) for $5 \mathrm{~min}$ three times, and incubated with secondary antibody at room temperature for $1 \mathrm{~h}$. Three 5-min PBST washes were then performed, followed by re-fixation with $4 \%$ paraformaldehyde (15713, Electron Microscopy Sciences) in PBS at room temperature for $10 \mathrm{~min}$ and two 5-min washes in PBS. Cells were then dehydrated by sequential incubation with $70 \%, 80 \%, 90 \%$, and $100 \%$ ethanol for $3 \mathrm{~min}$ and airdried. RNA-FISH was performed following immunostaining with Xist probes [40 ng of nick-translated pSx9-3 plasmids or 2 ng Xist oligonucleotide probes (see Supplementary Data 3 for sequences of FISH probes $\left.{ }^{67}\right]$. Images were acquired with a Nikon Eclipse 90i microscope and a Hamamatsu CCD camera. Image analysis was performed using Volocity (Perkin-Elmer)

In situ Hi-C. In situ Hi-C was performed following published protocols 6,28 using HindIII (100 U/ul; R0104M, NEB) and biotin-14-dATP (19524016, Thermo Fisher Scientific) on one WT and one Smchd1 $1^{-/-}$MEF clone, either without treatment, or transfected with scramble or HNRNPK- or RING1A- and RING1B-targeting small interfering RNA (siRNA) (see below for details of siRNA KD). We also performed in situ Hi-C on Xi ${ }^{A X i s t}$ fibroblasts. Hi-C libraries were sequenced on Illumina HiSeq, generating 60-150 millions paired-end 50 nucleotide reads per sample.

In situ Hi-C analysis. Each end of the Hi-C read pairs were aligned separately as single-end reads to the $129 \mathrm{~S} 1 / \mathrm{SvJm}$ (mus) and CAST/Eih (cas) genome using NovoAlign, followed by assignment of the allelic origin ${ }^{28}$. Alignments of the two ends were then joined. Allele-specific Hi-C contact matrices were constructed using Homer. Iterative correction of Hi-C matrices and construction of Pearson's correlation matrices at $200-\mathrm{kb}$ resolution were performed using HiTC. We noticed that the intensity of Pearson's correlation maps is influenced by sequencing depth. Therefore, we randomly down-sampled $\mathrm{Hi}-\mathrm{C}$ data to match the sequencing depth. Depth-corrected Hi-C contact maps and Pearson's correlation maps at 200-kb resolution were displayed in the manuscript. Unmappable regions, defined as bins with low read coverage (bottom $\sim 14 \%$ ), were set to NA before iterative correction. PCA was performed on Hi-C data without downsampling using Homer at 200-kb resolution, and the principal component profiles were visualized using IGV or R. To estimate the number and the average size of chromosome compartments, compartments were called using Homer at $100-\mathrm{kb}$ resolution in combination with custom R script. We noted that the replicate 2 of in situ $\mathrm{Hi}-\mathrm{C}$ in $\mathrm{Xi}^{\Delta X i s t}$ fibroblasts have more PCR duplicates than other samples. Therefore, this dataset was not further down-sampled and only used to calculate inter-megadomain interactions.

To calculate the correlation between compartment profiles and various chromatin features presented in Fig. 1c, we binned the X chromosome at $200 \mathrm{~kb}$ and computed Pearson's correlation coefficients, with unmappable regions excluded. H3K4me3 ChIP-seq (GSE33823), H2AK119ub ChIP-seq (GSE107217), H3K27me3 ChIP-seq (GSE36905), Xist CHART-seq (GSE48649), and SMCHD1 DamID-seq (GSE99991) datasets in the EY.T4 WT MEF line were downloaded from Gene Expression Omnibus (GEO). For Xist CHART-seq, H3K27me3 ChIPseq, and H2AK119ub ChIP-seq, we used input-subtracted coverage profiles generated by SPP with smoothing using 1-kb windows recorded every $500 \mathrm{bp}^{43}$

Insulation scores were generated from iteratively corrected $100-\mathrm{kb}$ binned $\mathrm{Hi}-\mathrm{C}$ matrices using the script matrix2insulation.pl (publically available on Github: https://github.com/dekkerlab/giorgetti-nature-2016) ${ }^{22}$, with parameters -is 500000 -ids 400000 -im iqrMean -nt 0 -ss 200000. For TADs and TAD boundaries, we used the coordinates identified from mouse embryonic stem cells defined previously ${ }^{2}$. To investigate the relationships between $\mathrm{S} 1 / \mathrm{S} 2$ compartments and TAD boundaries, we compared the PC1 values of Smchd1 $1^{-/}$MEF Xi computed at $100-\mathrm{kb}$ resolution with insulation profiles generated at the same resolution.

To compare the compartment profiles of MEFs with those of cells undergoing de novo XCI, we analyzed previously published Hi-C data of undifferentiated female ES cells (D0 ES) and embryoid bodies (EBs) formed after 4 or 7 days of differentiation (D4 and D7 EB) (GSE99991) ${ }^{28}$. We performed PCA on these datasets at 200-kb resolution using Homer. The hierarchical clustering analyses using the Euclidean or Pearson's correlation distance on compartment profiles were performed using the hclust function in $\mathrm{R}$.

To quantify the strength of megadomains, we computed \%inter-megadomain interactions, defined as the fraction of long-range chromatin interactions $(>10 \mathrm{Mb}$, approximately twice the size of the largest TAD on the X chromosome) that span the megadomain boundary $(73 \mathrm{Mb}, \mathrm{mm} 9)$ on depth-corrected Hi-C contact maps at $200-\mathrm{kb}$ resolution. When biological replicates were available, the fraction of inter-megadomain interactions in two biological replicates were computed separately and presented the means and standard deviation as bar plots. For the inter-megadomain interactions of WT MEFs plotted in Figs. 5e, 6e, and 7f, Hi-C data of WT MEF clone without treatment and WT MEFs transfected with scramble siRNA were considered as biological replicates. For the inter-megadomain interactions of Smchd1 $1^{-/}$MEFs plotted in the left panel of Fig. 6e, Hi-C data of Smchd1 $1^{-/-}$MEFs without treatment and Smchd1 ${ }^{-/-}$MEFs transfected with scramble siRNA (replicate 1) were considered as biological replicates.

RNA-sequencing. Total RNA was extracted from two WT and two Smchd1 $1^{-/-}$ clones treated with either DMSO or Aza using TRIzol (15596018, Thermo Fisher Scientific). Following ribosomal RNA depletion using RiboMinus Eukaryote Kit v2 (A15020, Thermo Fisher Scientific), strand-specific RNA-seq libraries were prepared $^{28}$. All libraries were sequenced with Illumina HiSeq, generating 32-63 millions paired-end 50 nucleotide reads per sample.

RNA-seq analysis. RNA-seq reads were aligned to the $129 \mathrm{~S} 1 / \mathrm{SvJm}$ (mus) and CAST/Eih (cas) genome using Tophat2, followed by assignment of the allelic origin $^{28}$. After removing PCR duplicates, unique exonic reads mapped to each gene were quantified by Homer. To compare the fold changes of autosomal and Xlinked genes, we analyzed only genes with fragments per kilobase of transcript per million mapped exonic reads (FPKM) $>0.1$. RNA-seq data of neural progenitor cells derived from WT or Smchd1 $1^{-1}$ female mouse embryonic stem cells (GSE99991) ${ }^{28}$ were downloaded from GEO. For allele-specific analysis, we defined \%mus as the percentage of mus-specific exonic reads in all allele-specific (musspecific + cas-specific) exonic reads of each transcript. To classify X-linked genes 
a

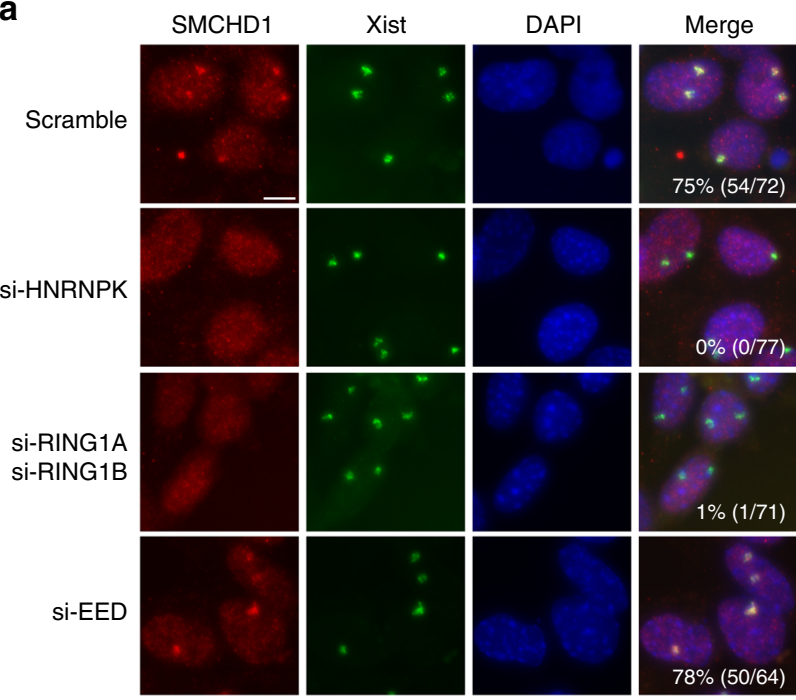

d

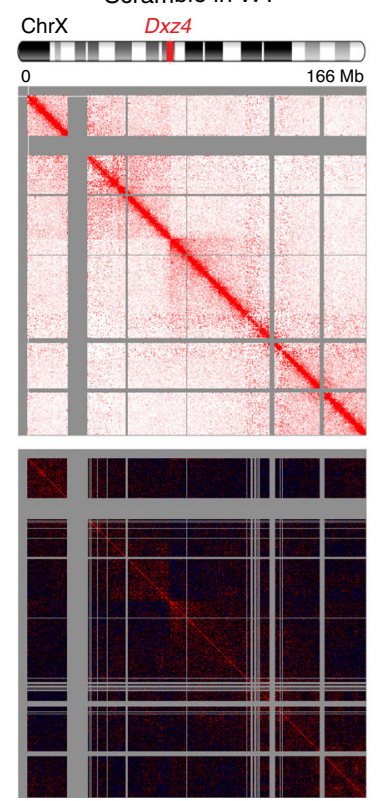

e
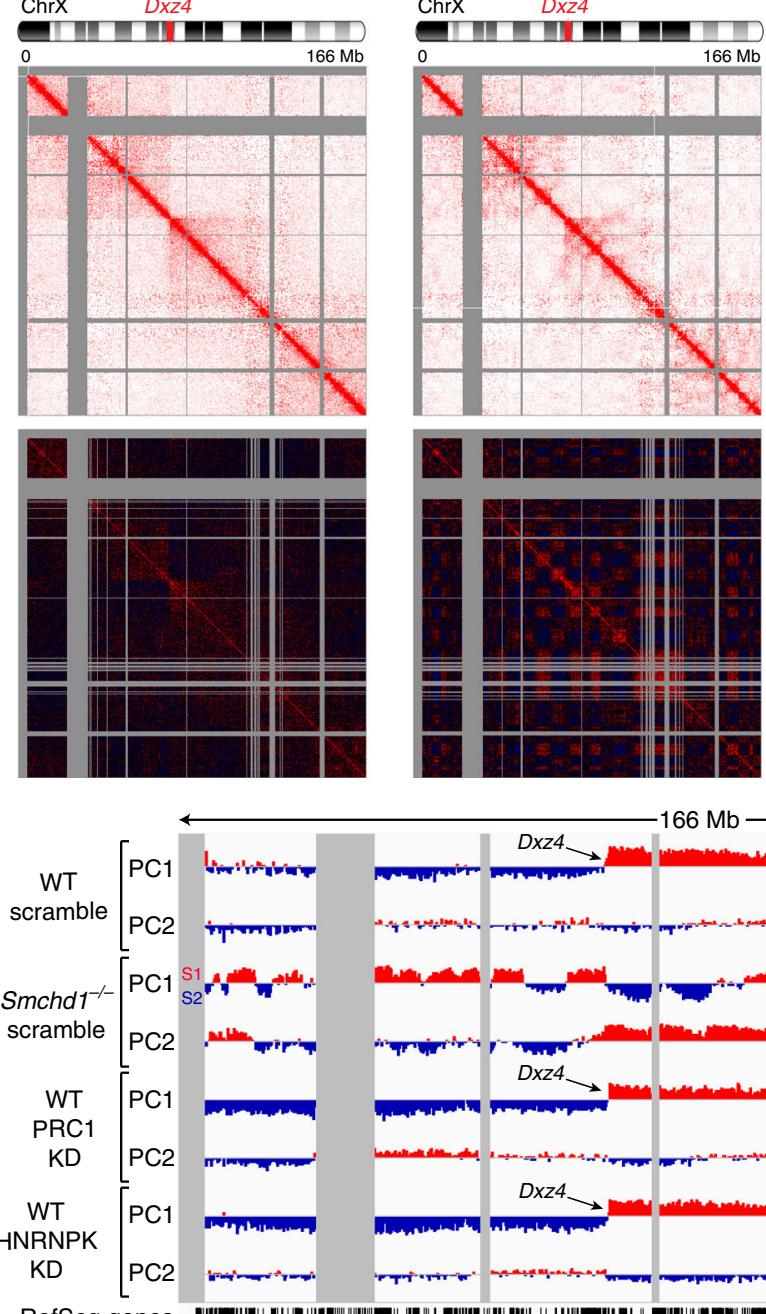

b
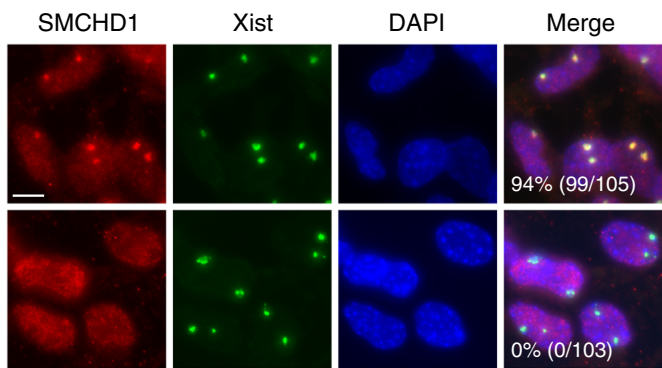

C
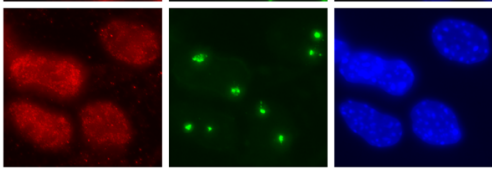

$0 \%(0 / 103)^{*}$
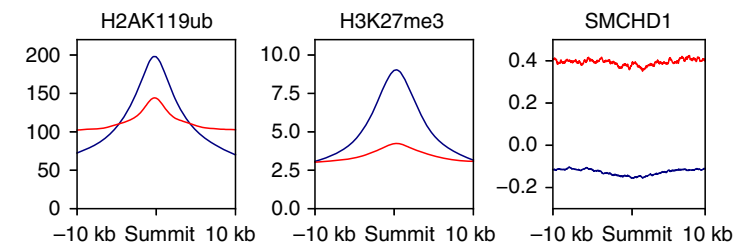

- Autosome - ChrX

PRC1 KD in WT

ChrX Dxz4
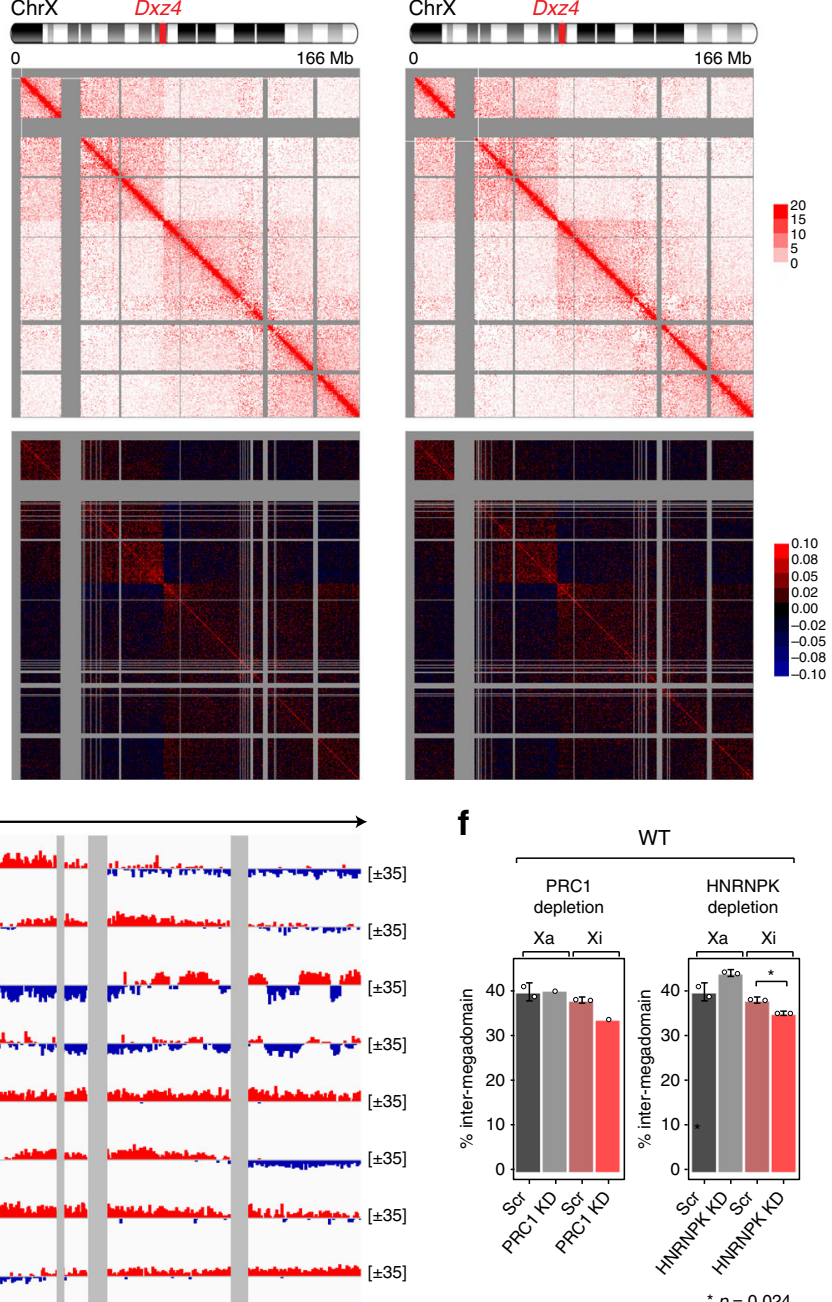

we defined active genes as genes with non-zero FPKM in all samples. Alleleassessable genes were defined as active genes with at least 13 allele-specific reads in all samples. A small fraction of genes overlap with incorrectly annotated singlenucleotide polymorphisms (SNPs) and produce unexpected allelic skewing, which were identified by analyzing the RNA-seq datasets of tail-tip fibroblasts (TTF) (GSE58524) and MEFs of pure Mus castaneous background ${ }^{66}$. Allele-assessable genes having \% mus $>9.09 \%$ in either pure cas TTFs or MEFs were considered as genes with miscalled SNPs. After excluding these genes, we defined escapees as active and allele-assessable genes whose expression from the $\mathrm{Xi}$ is $>10 \%$ of the expression from the $\mathrm{Xa}$ in at least one WT MEF clone. Genes subject to Xinactivation were defined as active and allele-assessable genes that were not genes with miscalled SNPs or escapees. Reactivated genes were defined as genes subject to XCI whose average \%mus in two Smchd1 $1^{-1}$ clones treated with Aza was (1) 3 -fold greater and (2) greater with the associated $p$ value $<0.1$ (one-sided $t$ test) than the 
Fig. 7 Heterogeneous nuclear ribonucleoprotein $\mathrm{K}$ (HNRNPK) and polycomb repressive complex 1 (PRC1) mediate structural maintenance of chromosomes hinge domain containing 1 (SMCHD1) recruitment and the stepwise folding of the inactive $\mathrm{X}$ chromosome (Xi). a Immuno-RNA-fluorescent in situ hybridization (immuno-RNA-FISH) for SMCHD1 and Xist on female wild-type mouse embryonic fibroblasts (WT MEFs) treated with small interfering RNA (siRNA) targeting HNRNPK, PRC1, and PRC2. Number of cells with SMCHD1 foci co-localizing with Xist clouds in a representative biological replicate is shown. Scale bar, $10 \mu \mathrm{m}$. b Immuno-RNA-FISH for SMCHD1 and Xist on female WT MEFs treated with dimethyl sulfoxide (DMSO) or MG132. Number of cells with SMCHD1 foci co-localizing with Xist clouds in a representative biological replicate is shown. Scale bar, $10 \mu \mathrm{m}$. c Chromatin binding profiles of H2AK119ub (GSE107217), H3K27me3 (GSE33823), and SMCHD1 (GSE99991) in female wild-type MEFs averaged over each 20-kb region centered at the summit of a H2AK119ub peak. d Depth-corrected chromatin interaction maps of the Xi in WT MEFs treated with control (scramble) siRNA, Smchd1 ${ }^{-/-}$ MEFs treated with control siRNA, and WT MEFs treated with RING1A/RING1B (PRC1 knockdown (KD)) or HNRNPK (HNRNPK KD) siRNA binned at 200$\mathrm{kb}$ resolution (top) and the corresponding Pearson's correlation maps (bottom). Gray-shaded areas, unmappable regions. Also see Supplementary Fig. 13a for the active X chromosome (Xa) maps. e Principal component 1 (PC1) and PC2 values of the Xi. Regions with positive PC1 values represent the S1 compartment or the telomeric megadomain. Gray-shaded areas, unmappable regions. Also see Supplementary Fig. 13b for PCs of the Xa. $\mathbf{f}$ Bar plots displaying the fraction of long-range interactions ( $>10 \mathrm{Mb}$ ) that span the Dxz4 megadomain boundary ("inter-megadomain" interactions) under different conditions. For HNRNPK KD, two replicates were analyzed, with $P$ values determined by the $t$ test (unpaired, one-sided). Error bars, s.d.

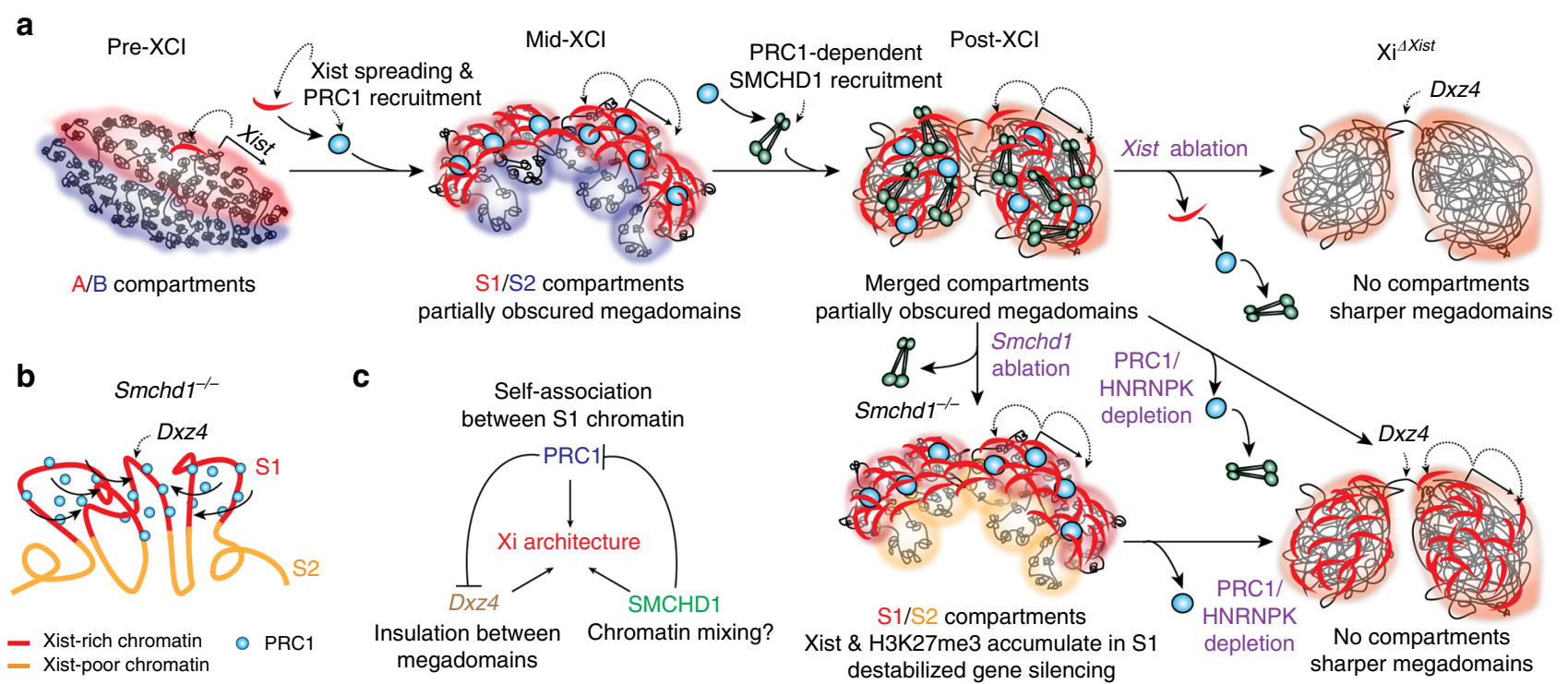

Fig. $8 \mathrm{~A}$ model for the folding and unfolding of the inactive $X$ chromosome (Xi) origami. a Summary: A stepwise folding process for the transformation of the Xi ("Origami Model"). Xist RNA is produced from the A compartment. Following transcription, Xist initially spreads to co-segregated A-compartmental chromatin. Through recruiting polycomb repressive complex 1 (PRC1), Xist reconfigures the $\mathrm{Xi}$ into S1/S2 compartments. Following structural maintenance of chromosomes hinge domain containing 1 (SMCHD1) recruitment (via a mechanism requiring heterogeneous nuclear ribonucleoprotein $K$ (HNRNPK), PRC1, and H2AK119ub), S1/S2 compartments are merged to form a compartment-less structure. In cells losing SMCHD1 after completing XCl, S1/S2 compartments reappear, coinciding with accumulation of Xist, H3K27me3, and H2AK119ub in the S1 compartment and destabilized gene silencing. Depleting PRC1 or HNRNPK diminishes the reappeared S1/S2 compartments, resulting in a "de-compartmentalized" Xi with sharper partition between the two megadomains. In cells that have undergone Xist ablation post XCl, both SMCHD1 and PRC1 fail to be recruited to the Xi, also leading to a "decompartmentalized" Xi with sharper megadomains. b Model: In the absence of SMCHD1, Xist-rich chromatin co-segregates, likely via PRC1 selfassociation, leading to the formation of S1/S2 compartments. The self-association between S1 chromatin creates long-range interaction across the Dxz4 boundary, thus partially obscuring megadomains. c Three major folding mechanisms that define the large-scale Xi architecture: self-association between Xist/H2AK119ub-enriched chromatin to form S1/S2 compartments through PRC1, potential chromatin mixing activity of SMCHD1, and inter-megadomain insulation by Dxz4. When superimposed on the Xi, these folding mechanisms sometimes interfere with each other-SMCHD1 attenuates S1/S2 structure, and PRC1 obscures megadomains

average \%mus in two WT clones treated with DMSO. To compare the cumulative distribution of \%mus of genes subject to XCI, we averaged the \%mus of two clones. To compare the effect of combined Smchd 1 ablation and Aza treatment on genes in S1 vs. S2 compartments, we defined the associated PC1 value of each X-linked gene as the $\mathrm{PC} 1$ value of the bin containing its transcriptional start site. Genes associated with positive PC1 values on the Smchd1 $1^{-1-} \mathrm{Xi}$ (100-kb resolution) were considered as residing in the S1 compartment. To investigate the effect of Smchd1 ablation and/or Aza treatment on the expression of escapees, we compared the \%mus of 11 genes defined as escapees in both WT clones.

We previously defined three classes (Class I-III) of genes subject to $\mathrm{XCI}^{28}$. Class I genes require SMCHD1 to be silenced during de novo XCI, and their gene bodies become depleted of the H3K27me3 mark in the absence of SMCHD1. Class II genes remain silent, enriched with $\mathrm{H} 3 \mathrm{~K} 27 \mathrm{me}$, but exhibit aberrant $\mathrm{H} 3 \mathrm{~K} 4 \mathrm{me} 3$ peaks at the promoter on the Xi. Class III genes are resistant to Smchd 1 ablation, with both the expression and the chromatin state (H3K4me3 and H3K27me3) unchanged in cells lacking SMCHD1. To investigate the difference between different classes of Xi genes in response to post-XCI Smchd1 ablation and Aza treatment, we intersected the 126 Class I genes with the 216 genes subject to XCI in MEFs. We then compared the cumulative distribution of the resulting 79 Class I genes and other 137 non-Class I genes. We noted that among the 216 genes subject to XCI, three genes (Rgag4, Timp1, and Slc10a3) were excluded from the categorization analysis in Wang et al. ${ }^{28}$, because they overlap with a longer gene. We assigned these three genes as "Class I" (Slc10a3), "Class III" (Rgag4), and "Unclassified" (Timp1), respectively, based on the criteria described previously ${ }^{28}$.

To visualize RNA-seq coverage, we generated strand-resolved fpm-normalized bigWig files from the raw RNA-seq reads for all reads (comp), mus-specific (mus) reads, and cas-specific (cas) reads separately, which were displayed using IGV with scales indicated in each track.

ChIP-seq of H3K27me3 and H2AK119ub. WT and Smchd1 $1^{-/}$MEFs were crosslinked in PBS with $1 \%$ formaldehyde at room temperature for 10 min at 2 million 
cells $/ \mathrm{ml}$ and quenched with $0.125 \mathrm{M}$ glycine at room temperature for $5 \mathrm{~min}$. Crosslinked cells were washed three times with chilled PBST ( $1 \times$ PBS with $0.05 \%$ Tween20) before snap-frozen in liquid nitrogen. Five million cross-linked cells were thaw on ice for $15 \mathrm{~min}$, resuspended in $2 \mathrm{ml}$ Buffer 1 [50 mM HEPES, pH 7.5, $150 \mathrm{mM}$ $\mathrm{NaCl}, 1 \mathrm{mM}$ EDTA, $0.5 \%$ Nonidet P-40, $0.2 \%$ Triton X-100, $1 \times$ complete EDTAfree Protease Inhibitor Cocktail (PIC; 11873580001, Sigma)], and rotated at $4^{\circ} \mathrm{C}$ for $10 \mathrm{~min}$. Nuclei were pelleted by centrifugation at $1700 \times g$ for $5 \mathrm{~min}$ at $4^{\circ} \mathrm{C}$, resuspended with $2 \mathrm{ml}$ Buffer $2(10 \mathrm{mM}$ Tris, $\mathrm{pH} 8.0,200 \mathrm{mM} \mathrm{NaCl}, 5 \mathrm{mM}$ EDTA, $2.5 \mathrm{mM}$ EGTA, $1 \times$ PIC) supplemented with $40 \mu \mathrm{l}$ Purelink RNaseA (12091021, Thermo Fisher Scientific), and incubated for $15 \mathrm{~min}$ with rotation at $4^{\circ} \mathrm{C}$. Nuclei were then pelleted again by centrifugation at $1700 \times g$ for $5 \mathrm{~min}$ at $4{ }^{\circ} \mathrm{C}$, resuspended with $1.5 \mathrm{ml}$ Buffer 3 (10 mM Tris, pH 8.0, 5 mM EDTA, 2.5 mM EGTA, $1 \times$ PIC) supplemented with $75 \mu \mathrm{l} 10 \% \mathrm{~N}$-lauroylsarcosine, and incubated for $15 \mathrm{~min}$ with rotation at $4{ }^{\circ} \mathrm{C}$. Following incubation, nuclei were transferred to five Evergreen Scientific polystyrene $1.5 \mathrm{ml}$ tubes $(250 \mu \mathrm{l}$ nuclear suspension per tube) and sonicated for $10 \mathrm{~min}$ using Qsonica Q800R3 (20 s ON/20 s OFF; amplitude 50\%). Sonicated chromatin was mixed at 1:1 ratio with $2 \times$ immunoprecipitation (IP) buffer $(30 \mathrm{mM}$ Tris, $\mathrm{pH} 8.0,300 \mathrm{mM} \mathrm{NaCl}, 2 \%$ Triton X-100, 0.5\% N-lauroylsarcosine, $1 \times$ PIC) and centrifuged at full speed for $30 \mathrm{~min}$ at $4{ }^{\circ} \mathrm{C}$ to remove insoluble debris. Two micrograms of chromatin was set aside as the input sample. For ChIP, $20 \mu \mathrm{g}$ chromatin was mixed with antibodies overnight with rotation at $4{ }^{\circ} \mathrm{C}$. Antibodies used were: $\alpha-\mathrm{H} 3 \mathrm{~K} 27 \mathrm{me} 3(2 \mu \mathrm{l}$; Millipore 07-449), $\alpha-\mathrm{H} 2 \mathrm{AK} 119 \mathrm{ub}$ ( $2 \mu$ l; Cell Signaling, 2899S), and normal rabbit IgG (2729S, Fisher Scientific), which served as a negative control. Following overnight incubation, chromatin was mixed with $20 \mu$ l Dynabeads Protein G (10004D, Thermo Fisher Scientific), which had been washed three times in $1 \times$ IP buffer $(15 \mathrm{mM}$ Tris, $\mathrm{pH} 8.0,150 \mathrm{mM} \mathrm{NaCl}$, $1 \%$ Triton X-100, 0.25\% $N$-lauroylsarcosine, $2.5 \mathrm{mM}$ EDTA, $1.25 \mathrm{mM}$ EGTA, $1 \times$ PIC), and incubated for $2 \mathrm{~h}$ with rotation at $4{ }^{\circ} \mathrm{C}$. Beads were then washed with $1 \mathrm{ml} 1 \times$ IP buffer once, $1 \mathrm{ml}$ wash buffer [50 mM HEPES, pH 7.5, $500 \mathrm{mM} \mathrm{LiCl}$, 1 mM EDTA, $1 \%$ Nonidet P-40, $0.7 \%$ sodium deoxycholate, $1 \times$ PIC] six times, and $1 \times$ TEN buffer (10 mM Tris, pH 8.0, $50 \mathrm{mM} \mathrm{NaCl}, 1 \mathrm{mM}$ EDTA) once. Following elution, crosslinks reversal, and validating ChIP quality, input and ChIP-enriched DNA were subject to library preparation using NEBNext ChIP-Seq Library Prep Master Mix Set for Illumina (E6240S, NEB) and sequenced on Illumina HiSeq, generating $\sim 30$ millions paired-end 50 nucleotide reads per sample. H3K27me3 ChIP-seq was performed in two biological replicates. Some of the samples were sequenced at the rapid-run mode on Illumina HiSeq to generate pair-end 69 nucleotide reads, which were trimmed to 50 nucleotides before downstream analysis.

Analysis of H3K27me3 and H2AK119ub ChIP-seq datasets. Input and ChIPseq reads were aligned to the $129 \mathrm{~S} 1 / \mathrm{SvJm}$ (mus) and CAST/Eih (cas) genome using NovoAlign, followed by assignment of the allelic origin ${ }^{28}$. After removing PCR duplicates, we generated fpm-normalized bigWig files from ChIP-seq reads for all uniquely mapped (comp), mus-specific (mus), and cas-specific (cas) reads separately, which were displayed using IGV with scales indicated in each track. As these two histone marks on the $\mathrm{X}$ chromosome originate predominantly from the $\mathrm{Xi}$, we displayed and used the comp tracks for analyses in the manuscript. The $\Delta \mathrm{H} 2 \mathrm{~A}-$ K119ub and $\Delta$ H3K27me3 tracks (Smchd1 $1^{-/}$minus WT) were computed by the bigwigCompare function of deepTools. H3K27me3 density at each X-linked genic and intergenic regions was computed by Homer, with the categorization of intergenic regions defined previously ${ }^{28}$. In addition to fpm normalization, we also generated input-subtracted ChIP tracks by SPP, with smoothing using 1-kb windows recorded every $500 \mathrm{bp}$, and fold-enrichment profiles of ChIP-seq over input using MACS2.

To compare the distribution of H2AK119ub and SMCHD1, we first used MACS2 $(q=0.01)$ to call summits of H2AK119ub. After filtering out peaks that mapped to ribosomal DNA and a false-positive region on chromosome 2 (chr2: 98,506,741-98,507,176, mm9), the coverage of H2AK119ub ChIP-seq (GSE107217), H3K27me3 ChIP-seq (GSE48649_MEF.K27me3.comp.bedGraph. gz), and SMCHD1 DamID-seq (GSM3036552_SMCHD1.rep1.DamID.comp.bw), at a $20-\mathrm{kb}$ region centered at each summit were computed and plotted as average profiles and heat maps by deeptools (v3.1.2).

Xist CHART-seq in WT and Smchd1 ${ }^{-/-}$MEFs. MEFs were cross-linked in PBS with $1 \%$ formaldehyde at room temperature for $10 \mathrm{~min}$ at 2 million cells $/ \mathrm{ml}$ and then quenched with $0.125 \mathrm{M}$ glycine at room temperature for $5 \mathrm{~min}$. Cross-linked cells were washed three times with chilled PBST ( $1 \times$ PBS with $0.05 \%$ Tween-20) before being snap frozen in liquid nitrogen at 25 million cross-linked cells per pellet. In total, two frozen pellets were used for each experiment. Nuclei were prepared following a published protocol ${ }^{28}$. Because of the larger volume of the nuclei in MEFs, nuclei were gradually resuspended in chilled sonication buffer (50 mM HEPES, pH 7.5, $75 \mathrm{mM} \mathrm{NaCl}, 0.1 \mathrm{mM}$ EGTA, 0.5\% $N$-lauroylsarcosine, $0.1 \%$ sodium deoxycholate, $5 \mathrm{mM}$ dithiothreitol, $10 \mathrm{U} / \mathrm{ml}$ SUPERaseIN) to reach approximately $270 \mu \mathrm{l}$ final volume. Nuclei were then sonicated in two $130 \mu \mathrm{l}$ bat ches within a Covaris microTUBE using Covaris E220e (140 W peak incident power, $10 \%$ duty factor, 200 cycles/burst). Sonicated chromatin was pooled and centrifuged at full speed for $20 \mathrm{~min}$ at $4{ }^{\circ} \mathrm{C}$. The supernatant $(\sim 220 \mu \mathrm{l})$ was then pooled to reach $\sim 440 \mu \mathrm{l}$ final volume, which was then split into two CHART reactions (Xist capturing CHART and a negative control using sense probes) following a protocol described previously (see Supplementary Data 3 for sequences of CHART probes $)^{28}$. CHART-enriched DNA was eluted by $20 \mu \mathrm{l}$ RNase $\mathrm{H}(5 \mathrm{U} / \mu \mathrm{l}$; M0297S, NEB) in $200 \mu \mathrm{l}$ elution buffer twice at $37^{\circ} \mathrm{C}$ for $30 \mathrm{~min}$. After validating CHART quality by quantitative PCR (qPCR) for the promoter of Xist and Gapdh (see Supplementary Data 3 for primer sequences), $100 \mu \mathrm{l}$ input and CHARTenriched DNA were mixed with $30 \mu \mathrm{l}$ TE buffer and further sheared to below 500 bp in a Covaris microTUBE for 2 min with Covaris E220e ( $140 \mathrm{~W}$ peak incident power, $10 \%$ duty factor, 200 cycles/burst). Sonicated DNA was purified and libraries were prepared by NEBNext ${ }^{\oplus}$ Ultra $^{\text {Dx }}$ DNA Library Prep Kit for Illumina ${ }^{\circledR}$ (E7370S, NEB). Experiments were performed in two biological replicates. Input and CHART-seq libraries were sequenced on Illumina HiSeq, generating 30 millions paired-end 50 nucleotide reads per sample.

Analysis of Xist CHART-seq datasets. Input and CHART-seq reads were processed as described in the section of ChIP-seq. After removing PCR duplicates, input-subtracted CHART profiles were generated using SPP, with smoothing using 1 -kb windows recorded every $500 \mathrm{bp}$. Because RNA-seq analysis showed that WT and Smchd1 ${ }^{-1-}$ MEFs expressed Xist at the same level, and Xist RNA-FISH did not detect a dispersed Xist cloud (indicative of a chromatin-attachment defect) in Smchd1 $1^{-/-}$cells, we extracted the X-chromosomal profiles from the Xist CHART profiles produced by SPP, and scaled the Xist coverage by equalizing the sum of Xist RNA coverage across the entire X chromosome in WT and Smchd1 ${ }^{-1-}$ MEFs using R. $\Delta$ Xist profiles were then generated by subtracting scaled WT profiles from those of Smchd1 $1^{-/-}$MEFs. Scaled Xist coverage profiles in WT and Smchd1 $1^{-/}$ MEFs and $\Delta$ Xist profiles were visualized using IGV, with scales indicated in each track. The density of Xist determined by CHART-seq at each X-linked genic and intergenic regions in WT or Smchd1 ${ }^{-1-}$ MEFs was computed by Homer on scaled CHART coverage profiles.

Western blot. Cells were washed once with PBS and lysed in chilled lysis buffer [ $10 \mathrm{mM}$ Tris, $\mathrm{pH} 8.0,1 \mathrm{M} \mathrm{NaCl}, 1 \%$ Triton, $0.5 \%$ sodium deoxycholate, $0.1 \%$ sodium dodecyl sulfate (SDS), $1 \mathrm{mM}$ EDTA, $0.5 \mathrm{mM}$ EGTA, $1 \times$ cOmplete EDTAfree PIC (11873580001, Sigma)]. Lysates were sonicated at $4{ }^{\circ} \mathrm{C}$ for $15 \mathrm{~min}(30 \mathrm{~s}$ ON; 30 s OFF) using Bioruptor ${ }^{\oplus} \mathrm{XL}$ (Diagenode). Proteins were quantified using Bio-Rad Protein Assay (500-0006, Bio-Rad). Lysates (20-50 $\mu \mathrm{g})$ were denatured in $1 \times$ Laemmli sample buffer at $95^{\circ} \mathrm{C}$ for 5 min and resolved by SDS-polyacrylamide gel electrophoresis. Proteins were transferred from the gels to PVDF membranes (Immobilon Transfer Membrane; IPVH00010, EMD Millipore) in 1× TBE in a TE77XP Semi-dry Blotter (Hoefer) at $0.8 \mathrm{~mA} / \mathrm{cm}^{2}$ for $45 \mathrm{~min}$. PVDF membranes were blocked with Blocking Buffer (5\% milk and $0.1 \%$ Tween-20 in PBS) at room temperature for $1 \mathrm{~h}$, incubated with antibodies against SMCHD1 (HPA039441, Sigma; 1:1000), RING1A (09-706, Millipore; 1:1000), RING1B (5694T, Cell Signaling; 1:1000), H2AK119ub (8240S, Cell Signaling; 1:2000), CTCF (2899S, Cell Signaling; 1:1000), or $\beta$-tubulin (T5201, Sigma, 1:2000) diluted in Blocking Buffer at $4{ }^{\circ} \mathrm{C}$ overnight. Membranes were then washed with PBST (PBS with $0.1 \%$ Tween-20) for $5 \mathrm{~min}$ three times, incubated with anti-rabbit IgG $(\mathrm{H}+\mathrm{L})$, HRP conjugate (W4011, Promega; 1:5000) or anti-mouse IgG (H + L), HRP conjugate (W4021, Promega; 1:10,000) diluted in Blocking Buffer at room temperature for 1 h, washed with PBST for 5 min three times, and developed using Western Lightning ${ }^{\oplus}$ Plus-ECL (NEL105001EA, Perkin-Elmer).

PRC1 and HNRNPK KD. One day prior to transfection, 0.6 million MEFs were plated on $10-\mathrm{cm}$ plates. The next day, cells were transfected with either scramble siRNA (siGENOME Non-Targeting siRNA \#1, D-001210-01, Dharmacon), siRNA targeting HNRNPK (L-048002-01, Dharmacon), or a mixture of siRNA targeting RING1A (D-062335-03, Dharmacon) and RING1B (D-042180-01, Dharmacon), using $30 \mu$ l Lipofectamine ${ }^{\circledast}$ RNAiMAX Transfection Reagent (13778075, Thermo Fisher Scientific). For HNRNPK KD, cells were transfected again after $24 \mathrm{~h}$ and harvested 3 days after the first transfection. For PRC1 KD, transfection was performed once a day for 4 days. Transfected cells were then used for in situ Hi-C, western blot, immuno-RNA-FISH, and quantitative reverse transcription-PCR (RT-qPCR).

Screening for protein factors required for SMCHD1 recruitment. To identify protein factors involved in recruiting SMCHD1 to the $\mathrm{Xi}$, we used siRNA to deplete several factors reported in three previous studies that systemically identified Xistinteracting proteins $s^{21,55,57}$. After identifying HNRNPK, we tested if PRC1 and PRC2, two Polycomb complexes targeted to the Xi via HNRNPK $24,55,56$, play a role in SMCHD1 recruitment. EY.T4 MEFs were transfected with either scramble control siRNA (D-001810-10-05, Dharmacon) or siRNA targeting HNRNPK (L048002-01, Dharmacon), SPEN (M-062019-01-0005, Dharmacon), LBR (GS98386, QIAGEN), and RBM15 (GS229700, Qiagen), EED (L-049898-00-0005, Dharmacon), RING1A (D-062335-03, Dharmacon), and RING1B (D-042180-01, Dharmacon) using Lipofectamine ${ }^{\circledast}$ RNAiMAX Transfection Reagent (13778075, Thermo Fisher Scientific). As a positive control, we treated cells with siRNA targeting SMCHD1 (L-040501-01-0005, Dharmacon). At $72 \mathrm{~h}$ after transfection, immuno-RNA-FISH for SMCHD1 and Xist was performed to determine the effect of siRNA treatment on SMCHD1 localization. We verified KD efficiency by western blots using rabbit polyclonal HNRNPK antibody (11426-1-AP, Proteintech, 
1:2000), rabbit polyclonal LBR antibody (12398-1-AP, Proteintech, 1:1000), rabbit polyclonal RBM15 antibody (ab70549, Abcam, 1:1000), rabbit polyclonal SMCHD1 antibody (HPA039441, Sigma, 1:1000), and rabbit monoclonal glyceraldehyde 3-phosphate dehydrogenase antibody (2118, Cell Signaling, 1:2000). KD of Ringla, Ringlb, Eed, and Spen was verified by RT-qPCR.

Quantitative reverse transcription-PCR. RNA was extracted using TRIzol (15596018, Thermo Fisher Scientific) according to the manufacturer's instructions. Genomic DNA was removed from RNA using TURBO DNA-free ${ }^{\text {rts }}$ Kit (AM1907, Thermo Fisher Scientific). After inactivating TURBO DNase by DNase Inactivation Reagent, DNase-treated RNA was reverse transcribed using SuperScript III Reverse Transcriptase (18080-085, Thermo Fisher Scientific) with 250 ng random primers (C118A, Promega) at $25^{\circ} \mathrm{C}$ for $5 \mathrm{~min}, 50^{\circ} \mathrm{C}$ for $1 \mathrm{~h}$, and $85^{\circ} \mathrm{C}$ for $15 \mathrm{~min}$. Complementary DNA was 5-10-fold diluted with $\mathrm{H}_{2} \mathrm{O}$. qPCR was then performed using iTaq ${ }^{\text {ma }}$ Universal SYBR ${ }^{\circledast}$ Green Supermix (1725125, Bio-Rad) in a CFX96 Real-Time PCR Detection System (Bio-Rad). To calculate the fraction of remaining transcripts in siRNA-treated cells, we normalized transcripts to $\beta$-actin, a housekeeping gene, and then set scramble siRNA-treated cells to $100 \%$. See Supplementary Data 3 for primer sequences.

Reporting summary. Further information on research design is available in the Nature Research Reporting Summary linked to this article.

\section{Data availability}

Raw and processed sequencing data have been deposited in the GEO accession GSE116413. The source data underlying Supplementary Figs. 1a, 11, 12a, 14b, and 14d are provided as a Source Data file. All other relevant data supporting the key findings of this study are available within the article and its Supplementary Information files or from the corresponding authors upon reasonable request.

\section{Code availability}

The custom analysis pipelines for all genomic analyses are available upon request with no restrictions.

Received: 27 March 2019 Accepted: 27 May 2019

Published online: 03 July 2019

\section{References}

1. Lieberman-Aiden, E. et al. Comprehensive mapping of long-range interactions reveals folding principles of the human genome. Science 326, 289-293 (2009).

2. Dixon, J. R. et al. Topological domains in mammalian genomes identified by analysis of chromatin interactions. Nature 485, 376-380 (2012).

3. Nora, E. P. et al. Spatial partitioning of the regulatory landscape of the Xinactivation centre. Nature 485, 381-385 (2012).

4. Nora, E. P. et al. Targeted degradation of CTCF decouples local insulation of chromosome domains from genomic compartmentalization. Cell 169 930-944 (2017). e22.

5. Rao, S. S. P. et al. Cohesin loss eliminates all loop domains. Cell 171, 305-320 (2017). e24

6. Rao, S. S. et al. A 3D map of the human genome at kilobase resolution reveals principles of chromatin looping. Cell 159, 1665-1680 (2014).

7. Sanborn, A. L. et al. Chromatin extrusion explains key features of loop and domain formation in wild-type and engineered genomes. Proc. Natl. Acad. Sci. USA 112, E6456-E6465 (2015).

8. Haarhuis, J. H. I. et al. The cohesin release factor WAPL restricts chromatin loop extension. Cell 169, 693-707 (2017). e14.

9. Schwarzer, W. et al. Two independent modes of chromatin organization revealed by cohesin removal. Nature 551, 51-56 (2017)

10. Larson, A. G. et al. Liquid droplet formation by HP1alpha suggests a role for phase separation in heterochromatin. Nature 547, 236-240 (2017).

11. Strom, A. R. et al. Phase separation drives heterochromatin domain formation. Nature 547, 241-245 (2017).

12. Plys, A. J. et al. Phase separation and nucleosome compaction are governed by the same domain of polycomb repressive complex 1. bioRxiv, https://doi.org/ $10.1101 / 467316$ (2018)

13. Tatavosian, R. et al. Nuclear condensates of the polycomb protein chromobox 2 (CBX2) assemble through phase separation. J. Biol. Chem. https://doi.org/ 10.1074/jbc.RA118.006620 (2018)

14. Pandya-Jones, A. \& Plath, K. The "Inc" between $3 \mathrm{D}$ chromatin structure and X chromosome inactivation. Semin Cell Dev. Biol. 56, 35-47 (2016).

15. Jegu, T., Aeby, E. \& Lee, J. T. The X chromosome in space. Nat. Rev. Genet. 18, 377-389 (2017).
16. Galupa, R. \& Heard, E. X-chromosome inactivation: a crossroads between chromosome architecture and gene regulation. Annu Rev. Genet. 52, 535-566 (2018).

17. Darrow, E. M. et al. Deletion of DXZ4 on the human inactive X chromosome alters higher-order genome architecture. Proc. Natl. Acad. Sci. USA 113, E4504-E4512 (2016).

18. Disteche, C. M. Dosage compensation of the sex chromosomes and autosomes. Semin. Cell Dev. Biol. 56, 9-18 (2016).

19. Froberg, J. E., Pinter, S. F., Kriz, A. J., Jegu, T. \& Lee, J. T. Megadomains and superloops form dynamically but are dispensable for X-chromosome inactivation and gene escape. Nat. Commun. 9, 5004 (2018).

20. Deng, $\mathrm{X}$. et al. Bipartite structure of the inactive mouse $\mathrm{X}$ chromosome. Genome Biol. 16, 152 (2015).

21. Minajigi, A. et al. Chromosomes. A comprehensive Xist interactome reveals cohesin repulsion and an RNA-directed chromosome conformation. Science 349, https://doi.org/10.1126/science.aab2276 (2015).

22. Giorgetti, L. et al. Structural organization of the inactive $\mathrm{X}$ chromosome in the mouse. Nature 535, 575-579 (2016).

23. Splinter, E. et al. The inactive $\mathrm{X}$ chromosome adopts a unique threedimensional conformation that is dependent on Xist RNA. Genes Dev. 25, 1371-1383 (2011)

24. Colognori, D., Sunwoo, H., Kriz, A. J., Wang, C. Y. \& Lee, J. T. Xist deletional analysis reveals an interdependency between Xist RNA and polycomb complexes for spreading along the inactive X. Mol. Cell 74, 101-117 (2019). e10.

25. Blewitt, M. E. et al. SmcHD1, containing a structural-maintenance-ofchromosomes hinge domain, has a critical role in $\mathrm{X}$ inactivation. Nat. Genet. 40, 663-669 (2008).

26. Gendrel, A. V. et al. Smchd1-dependent and -independent pathways determine developmental dynamics of $\mathrm{CpG}$ island methylation on the inactive X chromosome. Dev. Cell 23, 265-279 (2012).

27. Nozawa, R. S. et al. Human inactive X chromosome is compacted through a PRC2-independent SMCHD1-HBiX1 pathway. Nat. Struct. Mol. Biol. 20, 566-573 (2013).

28. Wang, C. Y., Jegu, T., Chu, H. P., Oh, H. J. \& Lee, J. T. SMCHD1 merges chromosome compartments and assists formation of super-structures on the inactive X. Cell 174, 406-421 (2018).

29. Yildirim, E., Sadreyev, R. I., Pinter, S. F. \& Lee, J. T. X-chromosome hyperactivation in mammals via nonlinear relationships between chromatin states and transcription. Nat. Struct. Mol. Biol. 19, 56-61 (2012).

30. Bonora, G. et al. Orientation-dependent Dxz4 contacts shape the 3D structure of the inactive X chromosome. Nat. Commun. 9, 1445 (2018).

31. Bickmore, W. A. \& van Steensel, B. Genome architecture: domain organization of interphase chromosomes. Cell 152, 1270-1284 (2013).

32. Bonev, B. \& Cavalli, G. Organization and function of the $3 \mathrm{D}$ genome. Nat. Rev. Genet. 17, 661-678 (2016)

33. Dixon, J. R., Gorkin, D. U. \& Ren, B. Chromatin domains: the unit of chromosome organization. Mol. Cell 62, 668-680 (2016).

34. Pope, B. D. et al. Topologically associating domains are stable units of replication-timing regulation. Nature 515, 402-405 (2014).

35. Sakakibara, Y. et al. Role of SmcHD1 in establishment of epigenetic states required for the maintenance of the $\mathrm{X}$-inactivated state in mice. Development 145, https://doi.org/10.1242/dev.166462 (2018).

36. Gdula, M. R. et al. The non-canonical SMC protein SmcHD1 antagonises $\mathrm{TAD}$ formation and compartmentalisation on the inactive $\mathrm{X}$ chromosome. Nat. Commun. 10, 30 (2019).

37. Csankovszki, G., Nagy, A. \& Jaenisch, R. Synergism of Xist RNA, DNA methylation, and histone hypoacetylation in maintaining $\mathrm{X}$ chromosome inactivation. I. Cell Biol. 153, 773-784 (2001).

38. Carrette, L. L. G. et al. A mixed modality approach towards Xi reactivation for Rett syndrome and other X-linked disorders. Proc. Natl. Acad. Sci. USA 115 E668-E675 (2018)

39. Berletch, J. B., Yang, F., Xu, J., Carrel, L. \& Disteche, C. M. Genes that escape from X inactivation. Hum. Genet. 130, 237-245 (2011).

40. Balaton, B. P. \& Brown, C. J. Escape artists of the X chromosome. Trends Genet. 32, 348-359 (2016).

41. Jansz, N. et al. Smchd1 targeting to the inactive $\mathrm{X}$ is dependent on the XistHnrnpK-PRC1 pathway. Cell Rep. 25, 1912-1923 (2018). e9.

42. Zhao, J., Sun, B. K., Erwin, J. A., Song, J. J. \& Lee, J. T. Polycomb proteins targeted by a short repeat RNA to the mouse X chromosome. Science 322, 750-756 (2008).

43. Simon, M. D. et al. High-resolution Xist binding maps reveal two-step spreading during X-chromosome inactivation. Nature 504, 465-469 (2013).

44. Zhang, L. F., Huynh, K. D. \& Lee, J. T. Perinucleolar targeting of the inactive X during $S$ phase: evidence for a role in the maintenance of silencing. Cell 129 693-706 (2007).

45. Hnisz, D., Shrinivas, K., Young, R. A., Chakraborty, A. K. \& Sharp, P. A. A phase separation model for transcriptional control. Cell 169, 13-23 (2017). 
46. Eskeland, R. et al. Ring1B compacts chromatin structure and represses gene expression independent of histone ubiquitination. Mol. Cell 38, 452-464 (2010).

47. de Napoles, M. et al. Polycomb group proteins Ring1A/B link ubiquitylation of histone $\mathrm{H} 2 \mathrm{~A}$ to heritable gene silencing and $\mathrm{X}$ inactivation. Dev. Cell 7, 663-676 (2004).

48. Fang, J., Chen, T., Chadwick, B., Li, E. \& Zhang, Y. Ringlb-mediated H2A ubiquitination associates with inactive $\mathrm{X}$ chromosomes and is involved in initiation of X inactivation. J. Biol. Chem. 279, 52812-52815 (2004).

49. Plath, K. et al. Developmentally regulated alterations in Polycomb repressive complex 1 proteins on the inactive X chromosome. J. Cell Biol. 167, 1025-1035 (2004).

50. Schoeftner, S. et al. Recruitment of PRC1 function at the initiation of $\mathrm{X}$ inactivation independent of PRC2 and silencing. EMBO J. 25, 3110-3122 (2006).

51. Kundu, S. et al. Polycomb repressive complex 1 generates discrete compacted domains that change during differentiation. Mol. Cell 65, 432-446 (2017). e5.

52. Kim, C. A., Gingery, M., Pilpa, R. M. \& Bowie, J. U. The SAM domain of polyhomeotic forms a helical polymer. Nat. Struct. Biol. 9, 453-457 (2002).

53. Bantignies, F. et al. Polycomb-dependent regulatory contacts between distant Hox loci in Drosophila. Cell 144, 214-226 (2011).

54. Schoenfelder, S. et al. Polycomb repressive complex PRC1 spatially constrains the mouse embryonic stem cell genome. Nat. Genet. 47, 1179-1186 (2015).

55. Chu, C. et al. Systematic discovery of Xist RNA binding proteins. Cell 161, 404-416 (2015).

56. Pintacuda, G. et al. hnRNPK recruits PCGF3/5-PRC1 to the Xist RNA Brepeat to establish polycomb-mediated chromosomal silencing. Mol. Cell 68, 955-969 (2017). e10.

57. McHugh, C. A. et al. The Xist lncRNA interacts directly with SHARP to silence transcription through HDAC3. Nature 521, 232-236 (2015).

58. Dantuma, N. P., Groothuis, T. A., Salomons, F. A. \& Neefjes, J. A dynamic ubiquitin equilibrium couples proteasomal activity to chromatin remodeling. J. Cell Biol. 173, 19-26 (2006).

59. Beagrie, R. A. et al. Complex multi-enhancer contacts captured by genome architecture mapping. Nature 543, 519-524 (2017).

60. Zheng, M. et al. Multiplex chromatin interactions with single-molecule precision. Nature 566, 558-562 (2019).

61. Rowley, M. J. \& Corces, V. G. Organizational principles of 3D genome architecture. Nat. Rev. Genet. 19, 789-800 (2018).

62. Kohlmaier, A. et al. A chromosomal memory triggered by Xist regulates histone methylation in X inactivation. PLoS Biol. 2, E171 (2004).

63. Lengronne, A. et al. Cohesin relocation from sites of chromosomal loading to places of convergent transcription. Nature 430, 573-578 (2004).

64. Bell, A. C. \& Felsenfeld, G. Methylation of a CTCF-dependent boundary controls imprinted expression of the Igf2 gene. Nature 405, 482-485 (2000).

65. Jansz, N. et al. Smchd1 regulates long-range chromatin interactions on the inactive X chromosome and at Hox clusters. Nat. Struct. Mol. Biol. 25, 766-777 (2018).

66. Beliveau, B. J. et al. Single-molecule super-resolution imaging of chromosomes and in situ haplotype visualization using Oligopaint FISH probes. Nat. Commun. 6, 7147 (2015)
67. Del Rosario, B. C. et al. Genetic Intersection of Tsix and Hedgehog signaling during the initiation of X-chromosome inactivation. Dev. Cell 43, 359-371 (2017). e6.

68. Jegu, T. et al. Xist RNA antagonizes the SWI/SNF chromatin remodeler BRG1 on the inactive X chromosome. Nat. Struct. Mol. Biol. 26, 96-109 (2019).

\section{Acknowledgements}

We thank J.E. Froberg and A. Kriz for critical reading of the manuscript. We thank all lab members for intellectual support, U. Kim and the MGH Next Generation Sequencing Core, and M. Kuroda, S. Buratowski, and A. Gimelbrant for valuable advice during C-Y. W.'s thesis work. This work was supported by grants to J.T.L. from the National Institute of Health (RO1-GM090278), the Rett Syndrome Research Trust, and International Rett Syndrome Foundation, and Howard Hughes Medical Institute.

\section{Author contributions}

C.-Y.W. and J.T.L. conceived the project, analyzed the data, and wrote the paper. C.-Y.W. performed all experiments and bioinformatics analyses. D.C. and H.S. contributed H2AK119ub ChIP-seq datasets for wild-type female MEFs. D.W. assisted with RT-qPCR, genotyping, and microscopy.

\section{Additional information}

Supplementary Information accompanies this paper at https://doi.org/10.1038/s41467 019-10755-3.

Competing interests: J.T.L. is a co-founder of and a scientific advisor for Translate Bio and Fulcrum Therapeutics. The other authors declare no competing interests.

Reprints and permission information is available online at http://npg.nature.com/ reprintsandpermissions/

Peer review information: Nature Communications thanks the anonymous reviewer(s) for their contribution to the peer review of this work. Peer reviewer reports are available.

Publisher's note: Springer Nature remains neutral with regard to jurisdictional claims in published maps and institutional affiliations.

Open Access This article is licensed under a Creative Commons Attribution 4.0 International License, which permits use, sharing, adaptation, distribution and reproduction in any medium or format, as long as you give appropriate credit to the original author(s) and the source, provide a link to the Creative Commons license, and indicate if changes were made. The images or other third party material in this article are included in the article's Creative Commons license, unless indicated otherwise in a credit line to the material. If material is not included in the article's Creative Commons license and your intended use is not permitted by statutory regulation or exceeds the permitted use, you will need to obtain permission directly from the copyright holder. To view a copy of this license, visit http://creativecommons.org/ licenses/by/4.0/

(C) The Author(s) 2019 